\title{
Mirror Formation Control in the Vicinity of an Asteroid
}

\author{
Massimiliano Vasile*, Christie Alisa Maddock ${ }^{\dagger}$ and Gianmarco Radice R $^{\ddagger}$ \\ University of Glasgow, Glasgow, G12 8QQ, United Kingdom
}

\begin{abstract}
Two strategies are presented for the positioning and control of a spacecraft formation designed to focus sunlight onto a point on the surface of asteroid, thereby sublimating the material and ejecting debris creating thrust. In the first approach, the formation is located at artificial equilibrium points around the asteroid and controlled using the force from the solar radiation pressure. The second approach determines the optimal periodic formation orbits, subject to the gravitational perturbations from the asteroid, the solar radiation pressure and the control acceleration derived from a control law.
\end{abstract}

\section{Nomenclature}

$\alpha \quad$ Angle of reflection, rad

$\beta \quad$ Solar aspect angle, rad

$\epsilon \quad$ Angular position of the AEP at $f_{0}$

$\eta_{\text {eff }} \quad$ Efficiency

$\mu \quad$ Gravitational constant, $\mathrm{km}^{3} / \mathrm{s}^{2}$

$\omega \quad$ Argument of periapsis, $\mathrm{rad}$

$\Omega \quad$ Right ascension of the ascending node, rad

$\theta \quad$ True longitude $(f+\omega), \operatorname{rad}$

a Semi-major axis, $\mathrm{km} \mid \mathrm{AU}$

A Surface area, $\mathrm{km}^{2}$

c Speed of light in space, $299792.458 \mathrm{~km} / \mathrm{s}^{2}$

d Diameter, $\mathrm{km}$

e Eccentricity

$f \quad$ True anomaly, rad

$F \quad$ Force, $\mathrm{mN}=\mathrm{kg} \cdot \mathrm{km} / \mathrm{s}^{2}$

$h \quad$ Orbital momentum, $\mathrm{km}^{2} / \mathrm{s}$

$i \quad$ Inclination, rad

$\mathbf{k} \quad$ Set of Keplerian orbital elements, $[a, e, i, \Omega, \omega, f, M]$

$l_{f} \quad$ Focal length, m

$l_{\text {offset }}$ Difference along the focal axis between the focal point and the intersection of the aperture plane, $\% l_{f}$

$L \quad$ Length of the projection of the adaptable mirror onto the $y_{\mathrm{M}}$-axis

$m \quad$ Mass, $\mathrm{kg}$

$M \quad$ Mean anomaly, rad

$n \quad$ Mean angular motion, $\mathrm{rad} / \mathrm{s}$

$p \quad$ Semi-latus rectum, $\mathrm{km}$

$\mathbf{r}, r \quad$ Radius, $\mathrm{km} \mid \mathrm{AU}$

$S_{0} \quad$ Solar flux density at $1 \mathrm{AU}, 1367 \mathrm{~W} / \mathrm{m}^{2}$

$T$ Period, days

$t \quad$ Time, s

* Senior Lecturer, Space Advanced Research Team, Department of Aerospace Engineering, James Watt Building South, University of Glasgow, email: m.vasile@aero.gla.ac.uk, AIAA Senior Member.

$\dagger^{\dagger}$ Ph.D. Candidate, Space Advanced Research Team, email: c.maddock@aero.gla.ac.uk, AIAA Student Member.

${ }^{\ddagger}$ Senior Lecturer, Space Advanced Research Team, email: g.radice@aero.gla.ac.uk, AIAA Member. 


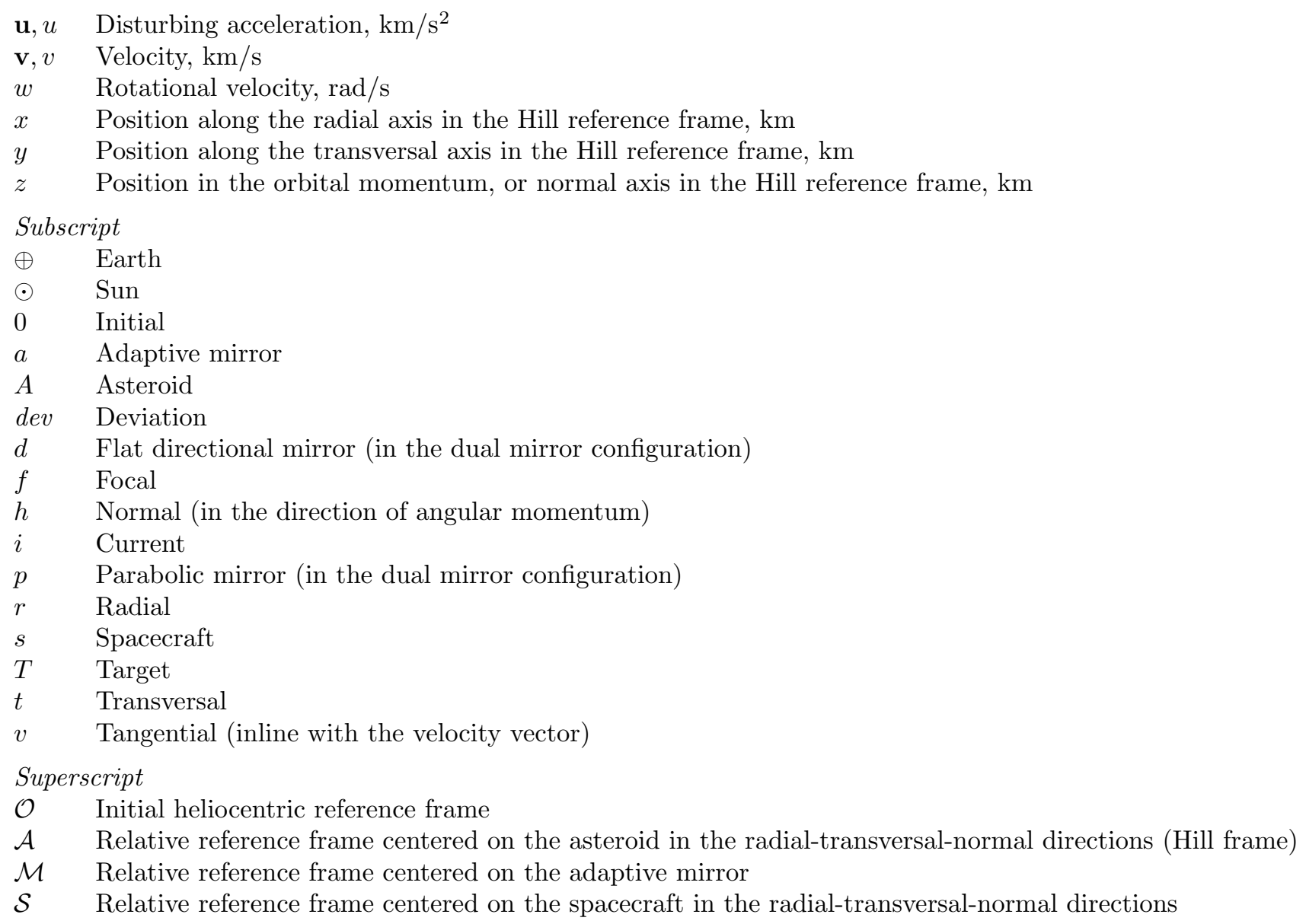

\section{Introduction}

$\mathrm{O}^{\mathrm{s}}$ VER the past decade, many different methods have been proposed for the deflection of asteroids, in particular those considered potentially hazardous. The methods range from kinetic or nuclear impactors, propulsive devices, induced changes to the asteroid surface and ablation devices. A study conducted by the team at the University of Glasgow ${ }^{1}$ compared the various deflection methods in terms of: achieved deviation distance, required warning time, total mass into orbit and the estimated technology readiness level.

A solar sublimation technique was found to be among the most effective methods. The idea was initially proposed $^{2}$ in 1992 and was compared a year later to other deflection methods by Melosh et al. ${ }^{3}$ The concept envisions a large mirror in space which would reflect sunlight onto the surface of the asteroid, sublimating the material and generating a low, continuous thrust due to the force of the ejected debris. However, the use of a single mirror would imply the deployment and control of a significantly large structure in space and presents a number of difficulties, as discussed recently by Kahle et al. ${ }^{4}$

In a recent study by the authors, ${ }^{5}$ it was demonstrated how a significant deviation of the asteroid Apophis could be achieved with a relatively small number of satellites (20 to 40) each carrying a relatively small primary mirror (between 10 and 40 meters in diameter). Fig. 1 shows a comparison of the required minimum diameter of the aperture of the primary parabolic mirror versus the duration of the thrust (see Fig. $2 a$ for the mirror configuration). The comparisons were done for different swarm sizes ranging from a single spacecraft (for a baseline comparison) up to 5000. For each swarm size, the diameter of the flat directional mirror was set to $0.5 \mathrm{~m}$ and $1.5 \mathrm{~m}$. The spot size on the surface is direct determined by the diameter and the angle of reflection of the collimated beam. The difference can be seen as the duration of the thrust increases; the lower branch corresponds to the $0.5 \mathrm{~m}$ diameter, and the upper branch, the $1.5 \mathrm{~m}$. This is expected as the higher the power density, the smaller the spot size (or a higher concentration ratio for the same incoming solar power). The deviation distance was nominally fixed equal to the Earth-Moon 


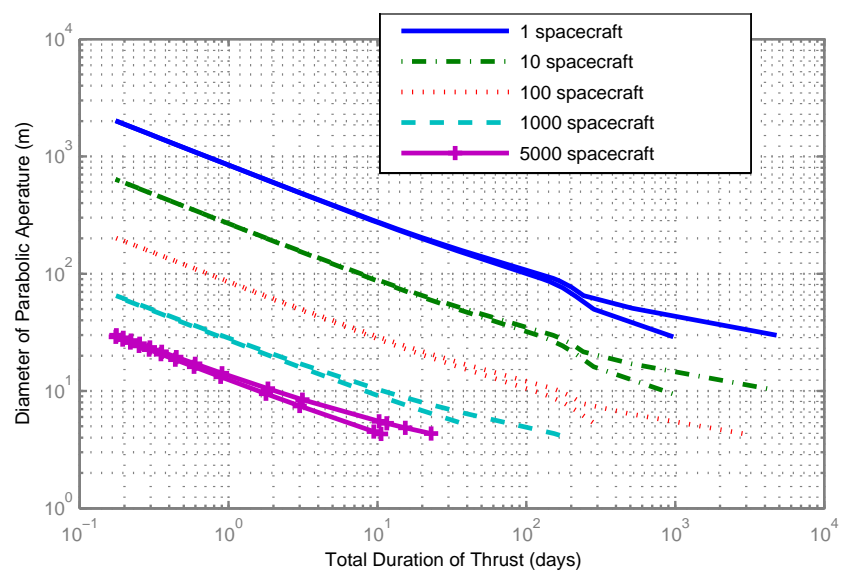

Figure 1: Diameter of the mirror vs. total duration of the deviation action for a variable number of spacecraft for a fixed deviation of the orbit of Apophis of $384403 \mathrm{~km}$ (Earth-Moon distance) at the MOID.

distance.

However, placing the mirrors in proximity of the asteroid was still an open issue. In particular the analysis of the orbital maintenance of the mirrors was still missing. In this paper, the multi-mirror option is presented together with an analysis of the positioning of the mirrors in the vicinity of the asteroid. A model for two different configurations - an innovative single and dual-mirror configurations - will be presented. Apophis is used as case study because of the relatively high threat posed by this particular asteroid.

\section{Physical Models}

The design of the device that is focusing the light of the Sun on the surface of the asteroid is a critical aspect of this deflection method. The device has to be able to concentrate a minimum power density at all times, ${ }^{6}$ therefore it is required to have the capability to steer the beam of light to hit any part of the asteroid and to control the concentration factor (or amount of light that is focused on a particular spot).

Here we propose two different configurations for the focusing device: a parabolic symmetric primary mirror with collimating lens and secondary directional mirror (Fig. 2a), and an asymmetric focusing mirror with collimating lens and no directional mirror (Fig. 2b). In the former case the primary mirror points always toward the Sun. The lens(es) produces a collimated beam of light that reflects on the secondary mirror and is projected onto the surface of the asteroid. In the latter case the primary mirror should be properly oriented based on the Sun vector. The configuration in Fig. $2 b$ can be easily modified by removing the lens and focusing the light directly on the surface of the asteroid. If the light is focused directly on the surface of the asteroid, the focal point has to be moved away from the mirror and the mirror will result to be almost flat.

\section{A. Single Mirror Configuration}

The single mirror configuration (see Fig. $2 b$ ) is composed of an asymmetric adaptive primary mirror and of a collimating lens (or set of lenses). The shape of the primary mirror is assumed to be adaptable such that the focal point can be moved in order to steer the beam in the desired direction.

If the mirror was flat we could define a local Cartesian reference frame with coordinated axes $\mathcal{M}\left[x_{\mathrm{M}}, y_{\mathrm{M}}, z_{\mathrm{M}}\right]$ centered in barycenter of the mirror assembly and with the $x \mathrm{M}$ perpendicular to the mirror surface (see Fig. 4). Now, we can define the shape of a curved mirror in the same reference frame. Given the position of the focal point in $\mathcal{M}$, the position of the center of a mirror element with infinitesimal area $d A$ and assuming a perfect reflection, the law of reflection gives us,

$$
\frac{d x_{\mathrm{M}}}{d y_{\mathrm{M}}}=\tan \left(\beta-\frac{\pi}{2}-\alpha\left(x_{\mathrm{M}}, y_{\mathrm{M}}, x_{f}, y_{f}, \beta\right)\right)
$$



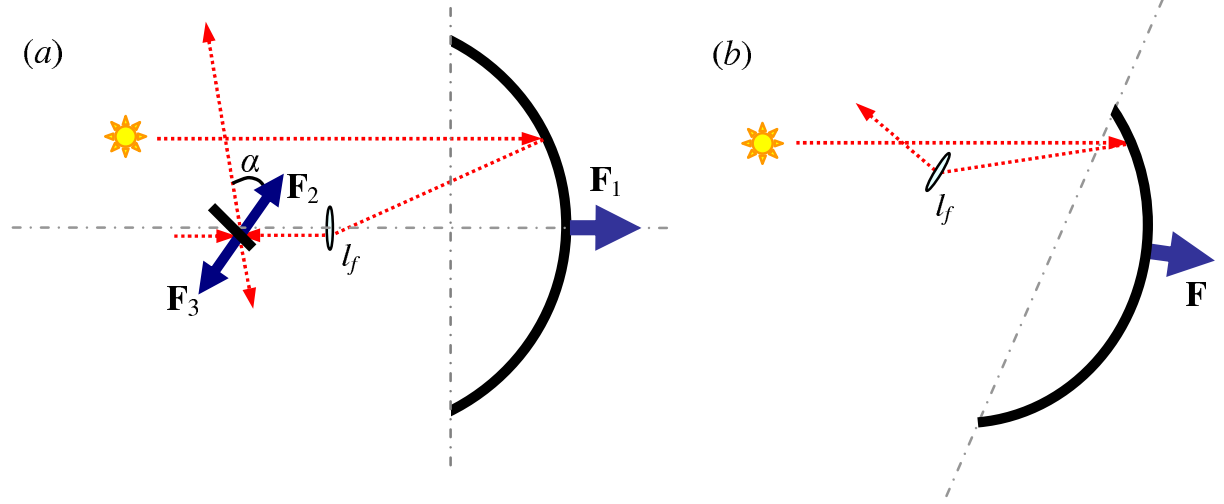

Figure 2: Different configurations for mirror assembly. ( $a$ ) fixed paraboloidic mirror with collimating lens and secondary directional mirror to steer the beam, $(b)$ concave mirror with adjustable focal point, and collimating lens.

where $\left[y_{f}, x_{f}\right]$ is the position of the focal point, $\beta$ is the Sun aspect angle with respect to the reference frame $\mathcal{M}$ of the mirror assembly and $\alpha$ is the reflection angle. Note that the angle $\beta$ also represents the attitude angle of the mirror reference frame with respect to the Hill reference frame $\mathcal{S}$ and therefore it will be referred to as the attitude angle of the mirror in the following. By integrating Eq. (1) with initial conditions $y_{\mathrm{M}_{0}}$ and $x_{\mathrm{M}_{0}}$, we can get the position and attitude of each section of the mirror in the $x_{\mathrm{M}}-y_{\mathrm{M}}$ plane given the position of the focal point and the direction of the incoming Sun rays. In the following, we will define the focal distance as $l_{f}=x_{f}-x_{\mathrm{M}_{0}}$. The mirror is then considered to be symmetric with respect to the $x_{\mathrm{M}}-y_{\mathrm{M}}$ plane such that each section of the mirror parallel to the $x_{\mathrm{M}^{-}} z_{\mathrm{M}}$ plane is a parabola with focus $l_{f}$.

Once the shape and orientation of the mirror are defined, the total force acting on the mirror assembly can be computed by integrating the following expression over the surface of the mirror $A_{a}$ :

$$
\mathbf{F}_{\mathrm{SRP}}=2 \eta_{\text {eff }} P_{r} \int_{A} \cos ^{2} \alpha \hat{\mathbf{n}} d A
$$

where $\eta_{\text {eff }}$ is the efficiency of the mirror (assumed to be 1.0), and $P_{r}$ is the solar pressure at a distance $r_{s}$ from the Sun given by,

$$
P_{r}=\frac{S_{0}}{c}\left(\frac{r_{\mathrm{AU}}}{r_{s}}\right)^{2}
$$

where $r_{\mathrm{AU}}$ is the distance at one Astronomical Unit (AU).

\section{B. Dual Mirror Configuration}

The dual mirror configuration (see Fig. $2 a$ ) consists of a parabolic reflector which concentrates the reflected sunlight onto a lens (or series of lenses) that collimate the beam. This beam is then directed onto the desired spot on the surface by means of a secondary, directional mirror. The primary parabolic mirror is held normal to the Sun to maximize the illuminated surface area, and hence power density on the surface.

The mirror is parabolic and symmetrical around the $z$-axis (i.e. the aperture is a circle). The illuminated surface area is calculated based on the focal length $l_{f}$ accounting for the blockage caused by the secondary mirror,

$$
\begin{aligned}
l_{f} & =\frac{\frac{1}{2} d_{p}}{2 \sqrt{l_{\text {offset }}}} \\
A_{p} & =\iint_{\frac{d_{d}}{2} \cos \alpha}^{\frac{d_{p}}{2}} 4 \sqrt{\left(\frac{x}{2 l_{f}}\right)^{2}+\left(\frac{y}{2 l_{f}}\right)^{2}+1} d x d y
\end{aligned}
$$




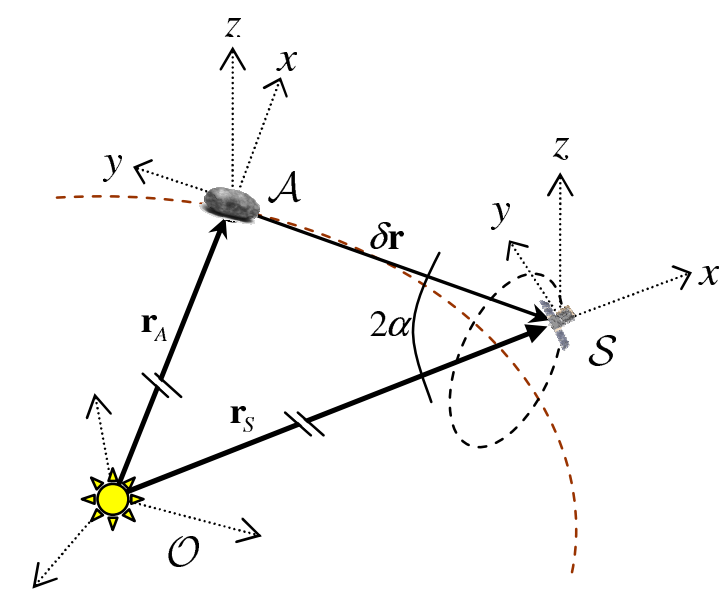

Figure 3: Definition of relative reference frames: $\mathcal{A}$ which is centered on the asteroid, and $\mathcal{S}$ which is centered on the spacecraft. Both are measured in radial $x$, transversal $y$ and normal $z$ directions.

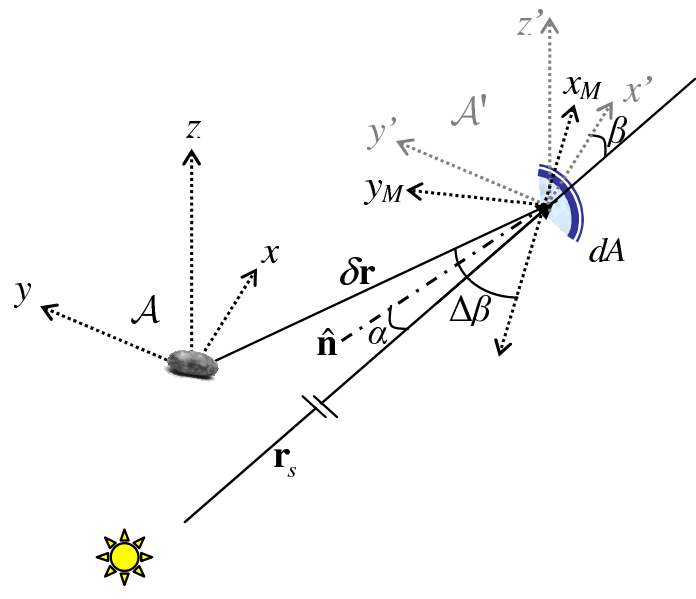

Figure 4: Definition of mirror-centric relative reference frame $\mathcal{M}$. The reference frame $\mathcal{A}^{\prime}$ is the translated from the barycenter of the asteroid to that of the mirror, with $\mathcal{A}^{\prime} \| \mathcal{A}$.

where $d_{p}$ is the diameter of the aperture on the parabolic mirror, $d_{d}$ is the diameter of the directional flat mirror and $\alpha$ is the angle of reflection. The depth of the mirror is given in terms of a percent of the focal length, $l_{\text {offset }}$ set here to $80 \%$. Clearly, the optimal depth would be equal to the focal length, however practically it is governed by the maximum allowable angle of incidence of the lens. It is also necessary to ensure that the flat mirror does not reflect the beam back onto any part of the parabolic mirror due to both blockage, and temperature concerns.

For the solar radiation pressure, three forces have to be taken into account: $\mathbf{F}_{1}$ is the force due to the solar pressure acting onto the primary mirror, $\mathbf{F}_{2}$ is the force due to the solar pressure acting onto the secondary mirror and $\mathbf{F}_{3}$ is the force due to the reflected light from the primary mirror onto the secondary mirror.

$$
\mathbf{F}_{2}=2 \eta_{\text {eff }} A_{p} P_{r} \hat{\mathbf{n}}
$$

Due to the concentration factor the power density of the reflected light is higher than the one of the direct light, therefore, though the surface of the secondary mirror is lower than the one of the primary, the reflected solar light exerts a force on the secondary mirror,

$$
\mathbf{F}_{3}=-\left(P_{r} \eta_{e f f_{p}} A_{p}\right) 2 \eta_{\text {eff }} \cos ^{2} \alpha \hat{\mathbf{n}}
$$

while $\mathbf{F}_{2}$ is simply

$$
\mathbf{F}_{2}=\left(2 \eta_{\text {eff }} \cos ^{2} \alpha\right) A_{d} P_{r} \hat{\mathbf{n}}
$$

Both forces are acting in the direction normal $\hat{\mathbf{n}}$ to the mirror surface $d A$.

The total force acting on the mirror assembly (i.e. primary, secondary mirror and lenses) is then given by,

$$
\mathbf{F}_{\mathrm{SRP}}=\mathbf{F}_{1}+\mathbf{F}_{2}+\mathbf{F}_{3}
$$

expressed as a vector in the local $\mathcal{S}$ Hill reference frame. In the following we will take into account only the contribution of the forces to the orbital dynamics but it should be noted that the composition of $\mathbf{F}_{2}$ and $\mathbf{F}_{3}$ will induce a consistent torque ont he mirror assembly.

\section{Asteroid Deflection Model}

From the initial observations, Apophis is expected to have a close encounter with the Earth in 2029. During that event Apophis could pass through a gravitational keyhole, a precise region in space no more than about 400 meters across, which would set up future resonant impacts starting on 13 April 2036. Table 1 give the orbital and physical data for the asteroid. ${ }^{7}$ 
Table 1: Estimated and observed orbital and physical properties of Apophis 99942.

\begin{tabular}{lll}
\multicolumn{1}{c}{ Element } & \multicolumn{2}{c}{ Measured Value } \\
\hline Semi-major axis & $a_{\mathrm{A}}$ & $0.9223 \mathrm{AU}$ \\
Eccentricity & $e_{\mathrm{A}}$ & 0.1911 \\
Inclination & $i_{\mathrm{A}}$ & $0.05814 \mathrm{rad}$ \\
Right ascension of the ascending node & $\Omega_{\mathrm{A}}$ & $3.5683 \mathrm{rad}$ \\
Argument of periapsis & $\omega_{\mathrm{A}}$ & $2.2059 \mathrm{rad}$ \\
Period & $T_{\mathrm{A}}$ & $323.50 \mathrm{days}$ \\
Mean motion & $n_{\mathrm{A}}$ & $2.2479 \mathrm{E}-7 \mathrm{rad} / \mathrm{s}$ \\
Mass $^{\text {a }}$ & $m_{\mathrm{A}}$ & $2.7 \mathrm{E}+10 \mathrm{~kg}$ \\
Physical dimensions $^{\mathrm{b}}$ & $a_{I}, b_{I}, c_{I}$ & $191 \mathrm{~m}, 135 \mathrm{~m}, 95 \mathrm{~m}$ \\
Rotational velocity & $w_{\mathrm{A}}$ & $5.8177 \mathrm{e}-5 \mathrm{rad} / \mathrm{s}$ \\
\hline
\end{tabular}

a The mass is estimated assuming a density of $2.6 \mathrm{~g} / \mathrm{cm}^{3}$.

$\mathrm{b}$ The physical dimensions estimated using an ellipsoidal model for the asteroid, based on the observed magnitude, where $a_{I} \leq b_{I} \leq c_{I}$ are the three radii along the three orthogonal axes.

The minimum orbital intersection distance (MOID) is defined as the separation distance at the closest point between two orbits, e.g. Apophis and the Earth. The deviation distance is defined here as the difference in $r_{\mathrm{A}}$ between the original, undeviated orbit and the deviated orbit at $t_{\mathrm{MOID}} \cdot{ }^{8}$ Non-linear equations were derived for determining the difference in $r_{\mathrm{A}}$ are expressed as a function of the ephemeris in the Hill reference frame $\mathcal{A}$ centered on the asteroid.

$$
\Delta \mathbf{r}_{d e v}=-\mathbf{r}_{\mathrm{A}}-\mathbf{r}_{\oplus}^{\mathcal{O}}\left[\begin{array}{c}
\varrho \cos \theta_{\mathrm{A}}+\zeta \sin \theta_{\mathrm{A}} \\
-\zeta \cos \theta_{\mathrm{A}}+\varrho \sin \theta_{\mathrm{A}} \\
-\cos \left(\Delta \theta-\theta_{\mathrm{A}}\right) \sin \Delta \Omega \sin i_{\mathrm{A}}+\varpi \sin \left(\Delta \theta-\theta_{\mathrm{A}}\right)
\end{array}\right]
$$

where

$$
\begin{aligned}
\varpi & =\cos i \sin (\Delta i-i)+\cos \Delta \Omega \cos (\Delta i-i) \sin i \\
\xi & =\cos \Delta \Omega \cos (\Delta i-i) \cos i-\sin (\delta i-i) \sin i \\
\varrho & =-\cos \Delta \Omega \cos (\Delta \theta-\theta)+\cos (\Delta i-i) \sin \Delta \Omega \sin (\Delta \theta-\theta) \\
\zeta & =\cos i \cos (\Delta \theta-\theta) \sin \Delta \Omega+\xi \sin (\Delta \theta-\theta)
\end{aligned}
$$

By definition of the coordinate system, $\mathbf{r}_{A}=\left[r_{A}, 0,0\right]^{T}$. The change in the orbital parameters are calculated by numerically integrating the Gauss planetary equations ${ }^{9}$ using a tangential thrust vector $\mathbf{u}_{d e v}$ induced by the sublimation method.

$$
\Delta k=\int_{t_{0}}^{t_{i}} \frac{d k\left(\mathbf{u}_{d e v}\right)}{d t} d t
$$

The change in angular location, in this case given by the mean anomaly, is calculated at the MOID by, ${ }^{8}$

$$
\Delta M=\int_{t_{0}}^{t_{i}} \frac{d M}{d t} d t+n_{A_{0}}\left(t_{0}-t_{\mathrm{MOID}}\right)+n_{A_{i}}\left(t_{\mathrm{MOID}}-t_{i}\right)
$$

where the mean motion is given by,

$$
n=\sqrt{\frac{\mu}{a^{3}}}
$$

The thrust produced by the deflection method is a direct function of the rate of the expelled surface matter, $\dot{m}_{\text {exp. }}{ }^{10}$

$$
\frac{d m_{\text {exp }}}{d t}=2 v_{\text {rot }} \int_{y_{0}}^{y_{\text {max }}} \int_{t_{\text {in }}}^{t_{\text {out }}} \frac{1}{E_{v}}\left(P_{\text {in }}-Q_{\text {rad }}-Q_{\text {cond }} \sqrt{\frac{1}{t}}\right) d t d y
$$


where $\left[t_{\text {in }}, t_{\text {out }}\right]$ is the duration for which the point is illuminated, $\left[y_{0}, y_{\max }\right]$ are the limits of the vertical illuminated surface area (i.e. orthogonal to the direction of rotation of the asteroid $v_{r o t}$ ), $E_{v}$ is the enthalpy of sublimation, $P_{i n}$ is the input power due to the solar concentrators, $Q_{\text {rad }}$ is the heat loss due to black-body radiation and $Q_{\text {cond }}$ is the conduction loss.

The magnitude of the induced acceleration can then determined by, ${ }^{10}$

$$
\mathbf{u}_{d e v}=\frac{\frac{2}{\pi} \bar{v} \dot{m}_{\exp }}{m_{A_{i}}} \cdot \hat{\mathbf{v}}_{\mathrm{A}}
$$

where $\hat{\mathbf{v}}_{\mathrm{A}}$ is direction of velocity vector of the NEO, $\left(\frac{2}{\pi}\right)$ is the scattering factor assuming the debris plume is uniformly distributed over a half-sphere, $\bar{v}$ is the average velocity of the debris particles according to Maxwell's distribution of an ideal gas, and the remaining mass of the asteroid $m_{A_{i}}$ is calculated by numerically integrating Eq. (14).

\section{Spacecraft Orbital Dynamics}

\section{A. Artificial Equilibrium Points}

If solar pressure and the gravity field of the asteroid are taken into account then the mirrors can be designed so that the two forces are in equilibrium, with the spacecraft hovering at a fixed location and distance from the asteroid, using the single-mirror configuration to control the beam. If we consider a perfectly spherical and homogenous gravity field of the asteroid, the dynamics of the mirror is governed by the following set of equations,

$$
\begin{aligned}
& \ddot{x}=2 \dot{f}\left(\dot{y}-y \frac{\dot{r}_{A}}{r_{A}}\right)+x \dot{f}^{2}+\frac{\mu_{\odot}}{r_{A}^{2}}-\frac{\mu_{\odot}}{\delta r^{3}}\left(r_{A}+x\right)-\frac{\mu_{A}}{\delta r^{3}} x+\frac{s_{x}(x, y, z)}{m_{s}}+\frac{u_{x}}{m_{s}} \\
& \ddot{y}=-2 \dot{f}\left(\dot{x}-x \frac{\dot{r}_{A}}{r_{A}}\right)+y \dot{f}^{2}-\frac{\mu_{\odot}}{r_{s}^{3}} y-\frac{\mu_{A}}{\delta r^{3}} y+\frac{s_{y}(x, y, z)}{m_{s}}+\frac{u_{y}}{m_{s}} \\
& \ddot{z}=-\frac{\mu_{\odot}}{r_{s}^{3}} z-\frac{\mu_{A}}{\delta r^{3}} z+\frac{s_{z}(x, y, z)}{m_{s}}+\frac{u_{z}}{m_{s}}
\end{aligned}
$$

where $m_{s}$ is the estimated mass of the spacecraft, $\mathbf{F}_{\mathrm{SRP}}=\left[s_{x}, s_{y}, s_{z}\right]$ is the solar force in Eq. (2) and $\mathbf{u}=\left[u_{x}, u_{y}, u_{z}\right]^{T}$ is the control force. We are now interested in solutions of the following system,

$$
\begin{aligned}
2 \dot{f}\left(-y \frac{\dot{r}_{A}}{r_{A}}\right)+x \dot{f}^{2}+\frac{\mu_{\odot}}{r_{A}^{2}}-\frac{\mu_{\odot}}{r_{s}^{3}}\left(r_{A}+x\right)-\frac{\mu_{A}}{\delta r^{3}} x+\frac{s_{x}\left(x, y, z, \beta, l_{f}\right)}{m_{s}} & =0 \\
-2 \dot{f}\left(-x \frac{\dot{r}_{A}}{r_{A}}\right)+y \dot{f}^{2}-\frac{\mu_{\odot}}{r_{s}^{3}} y-\frac{\mu_{A}}{\delta r^{3}} y+\frac{s_{y}\left(x, y, z, \beta, l_{f}\right)}{m_{s}} & =0 \\
-\frac{\mu_{\odot}}{r_{s}^{3}} z-\frac{\mu_{A}}{\delta r^{3}} z+\frac{s_{z}\left(x, y, z, \beta, l_{f}\right)}{m_{s}} & =0
\end{aligned}
$$

The third equation (17c) is always satisfied if the mirror is in the $x_{\mathrm{M}^{-}} y_{\mathrm{M}}$ plane therefore in the following we will focus on the motion in this plane. Now, considering that the mirror has to constantly reflect the light onto the surface of the asteroid, if the mirror is flat the only possible equilibrium configuration is with the asteroid-mirror direction aligned with the spacecraft-Sun direction. If the mirror is not flat, then we can look for possible position vector $\delta \mathbf{r}$, solar aspect angle $\beta$ and focal distance $l_{f}$ such that the vector $\mathbf{F}_{\mathrm{SRP}}$ is aligned with the asteroid-mirror direction.

Fig. 6 represents the misalignment of the force vector due to the solar pressure with respect to the spacecraft-asteroid direction. The angle $\beta$ is the direction of the light impacting on the mirror while $\Delta \beta$ is the angle between the incoming sunlight and the direction of the focal point of the mirror. The direction of the focal point identifies the pointing direction. We consider only one quadrant of the Hill frame with positive $x$ and negative $y$. For positive $x$ and positive $y$ the solutions are symmetric; there are no solutions in the other two quadrants.

As it can be seen for $\beta=\pi / 2$, the only artificial equilibrium points (AEP) are along the Sun-asteroid direction. However, in this case the mirror would be in shadow and therefore no equilibrium points can exist along that direction. For higher values of $\beta$, equilibrium points can exist at higher angular distances 
from the radial direction. For example, for $\beta=139^{\circ}$ the mirror can be placed at $\delta \mathbf{r}=[1.3699,0.48225,0]$ $\mathrm{km}$, which is about $20^{\circ}$ from the radial direction (see Fig. 5 shows the level of acceleration acting on the spacecraft).

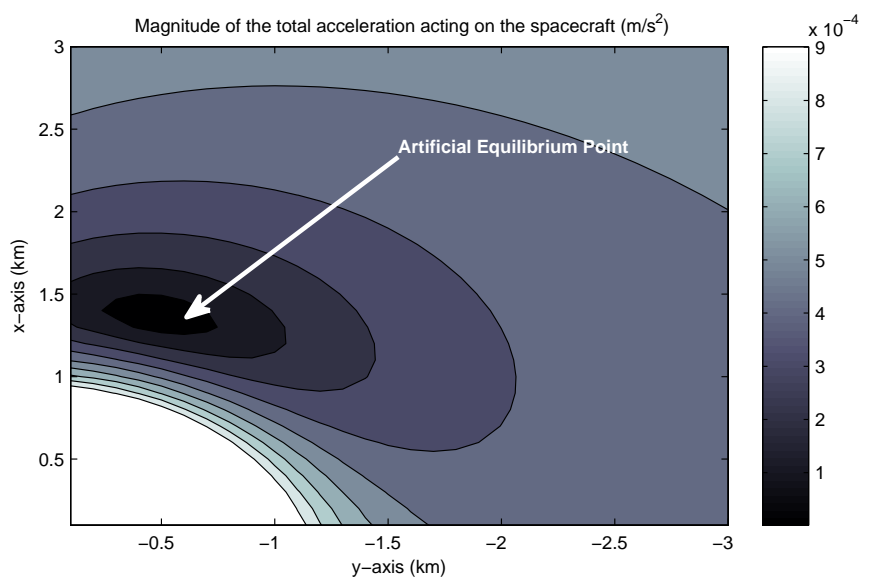

Figure 5: Example of AEP at $20^{\circ}$ from the radial direction.

This artificial equilibrium point offers a good location for projecting the light of the Sun on the side of the asteroid along the $y$ direction, and away from the plume of gases. If we assume that the lens produces a collimated light beam with negligible divergence, and that the beam is projected at the intersection of the surface of the asteroid with the $y$-axis, then we can compute where the two extreme points of the beam intersect the surface of the asteroid. From this intersection, we can compute the spot size given the beam size and the elevation over the $y$-axis. As can be seen in Fig. 8, for a beam size between 0.5 and $1 \mathrm{~m}$ in diameter, the increase in spot size due to an elevation of $70^{\circ}$ degrees along the $y$-axis and $20^{\circ}$ from the radial $x$-axis, is still limited.
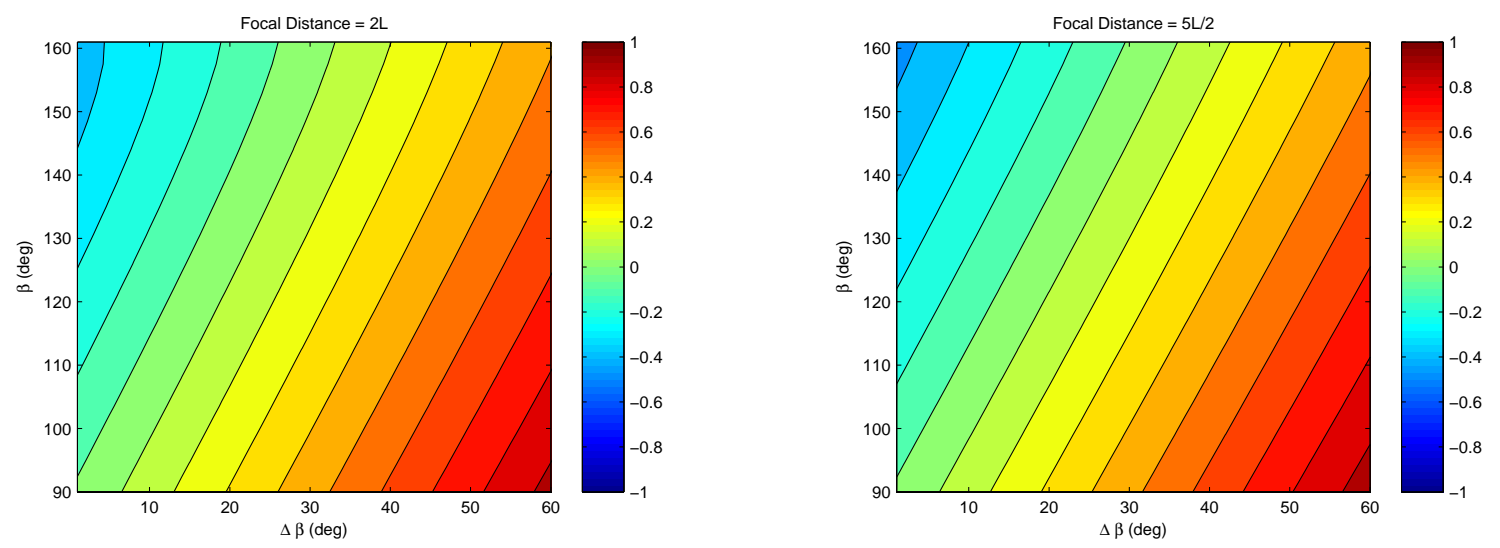

Figure 6: Misalignment between the position vector $\delta \mathbf{r}$ and the direction of resultant force due to solar pressure: (left) the misalignment for a focal distance equal to $2 L$; (right) the misalignment for a focal distance equal to $2.5 L$, where $L$ is the length of the projection of the mirror on the $y_{\mathrm{M}}$ axis.

It should be noted that due to the movement of the asteroid along its orbit the AEPs do not keep a fixed position since the modulus of the solar force is changing with the inverse of the square of the distance from the Sun. If we compute the AEP for every position of the asteroid along its orbit we get the result in Fig. 9.

These results are useful to identify AEPs in the case of a perfectly spherical asteroid. On the other hand, the actual shape of Apophis and of a general asteroid cannot be considered spherical, therefore consistent with studies by other authors, the asteroid was modeled as an ellipsoid with semi-axes $a_{I}, b_{I}$ and $c_{I}$ (see 


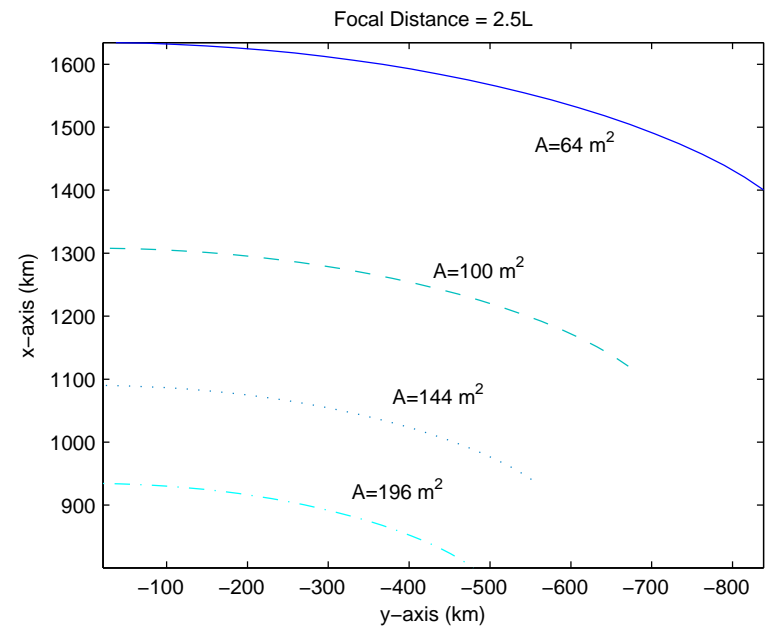

Figure 7: Artificial equilibrium points for different mirror sizes.

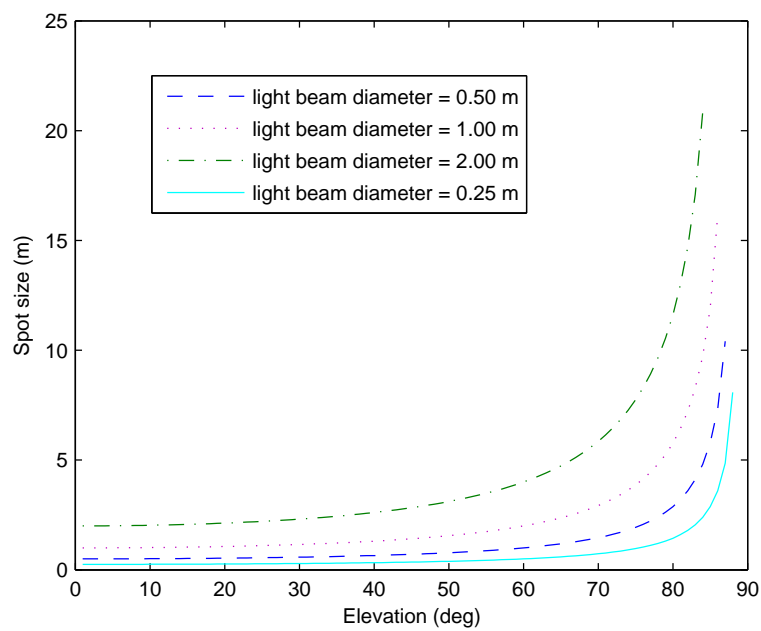

Figure 8: Increase in the spot size as a function of the elevation above the $y$ axis.
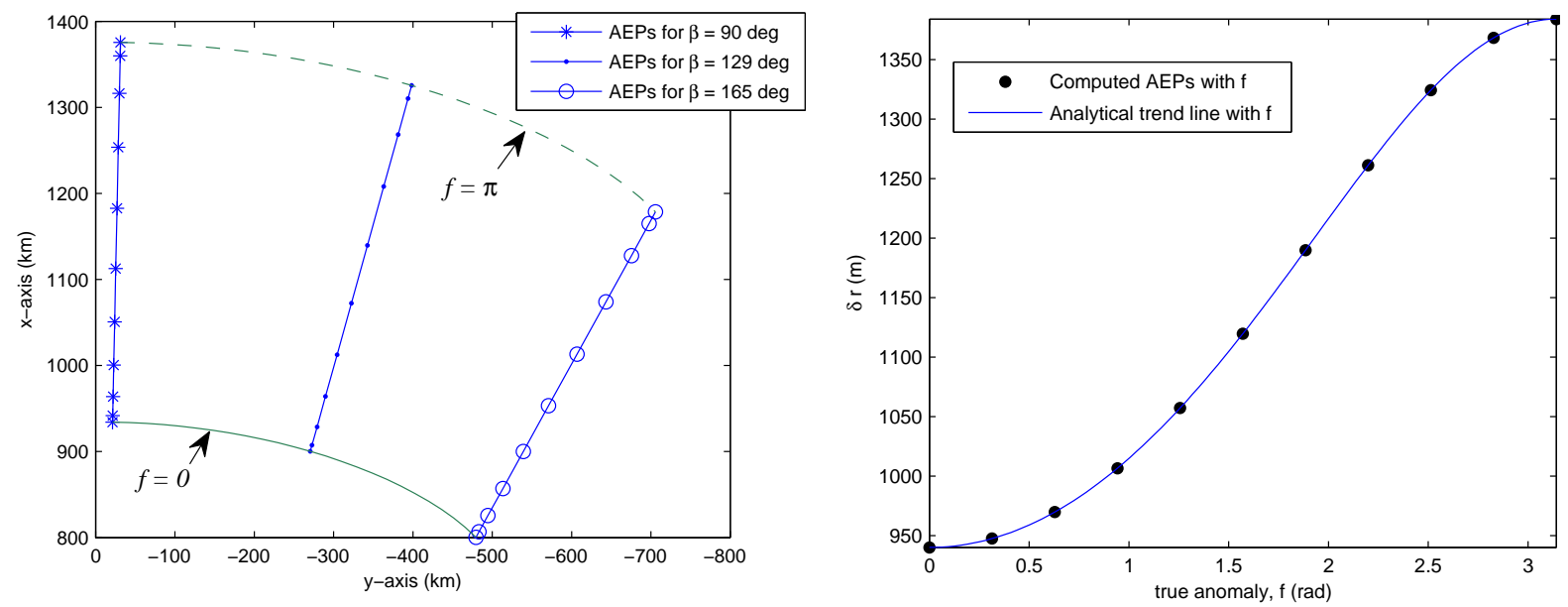

Figure 9: Variation of the equilibrium points with the true anomaly for $A=196 \mathrm{~m}^{2}$. 
Table 1). We assume that the semi-axis $c_{I}$ is aligned with the $z$-axis of the $\mathcal{A}$ Hill frame and that the asteroid is rotating around the $z$-axis with angular velocity $w_{A}$. The gravity field of the asteroid can be expressed as the sum of a spherical field plus a second-degree and second-order field, ${ }^{11,12}$

$$
U_{20+22}=\frac{\mu_{\mathrm{A}}}{\delta r^{3}}\left(C_{20}\left(1-\frac{3}{2} \cos ^{2} \gamma\right)+3 C_{22} \cos ^{2} \gamma \cos 2 \lambda\right)
$$

where the harmonic coefficients $C_{20}$ and $C_{22}$ can be expressed as a function of the semi-axes,

$$
\begin{aligned}
& C_{20}=-\frac{1}{10}\left(2 c_{I}^{2}-a_{I}^{2}-b_{I}^{2}\right) \\
& C_{22}=\frac{1}{20}\left(a_{I}^{2}-b_{I}^{2}\right)
\end{aligned}
$$

where $\lambda$ is defined as,

$$
\lambda=\arctan \left(\frac{y}{x}\right)+w_{A} t
$$

and $\gamma=0$ since we are only interested in the in-plane motion. The angular speed is assumed to be $w_{A}=5.8177 \mathrm{e}-5 \mathrm{rad} / \mathrm{s}$, or one revolution every 30 hours. ${ }^{10}$ Therefore, the equations for the orbital dynamics of the spacecraft can be expressed as,

$$
\begin{aligned}
2 \dot{f}\left(-y \frac{\dot{r}_{A}}{r_{A}}\right)+x \dot{f}^{2}+\frac{\mu_{\odot}}{r_{A}^{2}}-\frac{\mu_{\odot}}{r_{s}^{3}}\left(r_{A}+x\right)-\frac{\mu_{A}}{\delta r^{3}} x+\frac{s_{x}\left(x, y, z, \beta, l_{f}\right)}{m_{s}}+\frac{\partial U_{20+22}}{\partial x} & =0 \\
-2 \dot{f}\left(-x \frac{\dot{r}_{A}}{r_{A}}\right)+y \dot{f}^{2}-\frac{\mu_{\odot}}{r_{s}^{3}} y-\frac{\mu_{A}}{\delta r^{3}} y+\frac{s_{y}\left(x, y, z, \beta, l_{f}\right)}{m_{s}}+\frac{\partial U_{20+22}}{\partial y} & =0 \\
-\frac{\mu_{\odot}}{r_{s}^{3}} z-\frac{\mu_{A}}{\delta r^{3}} z+\frac{s_{z}\left(x, y, z, \beta, l_{f}\right)}{m_{s}}+\frac{\partial U_{20+22}}{\partial z} & =0
\end{aligned}
$$

Note that even in this case Eq. (20c) is satisfied for $z=0$. If the actual shape of the asteroid is considered the AEP position is not moving along a rectilinear line anymore but is following a spiralling path as in Fig. 10.
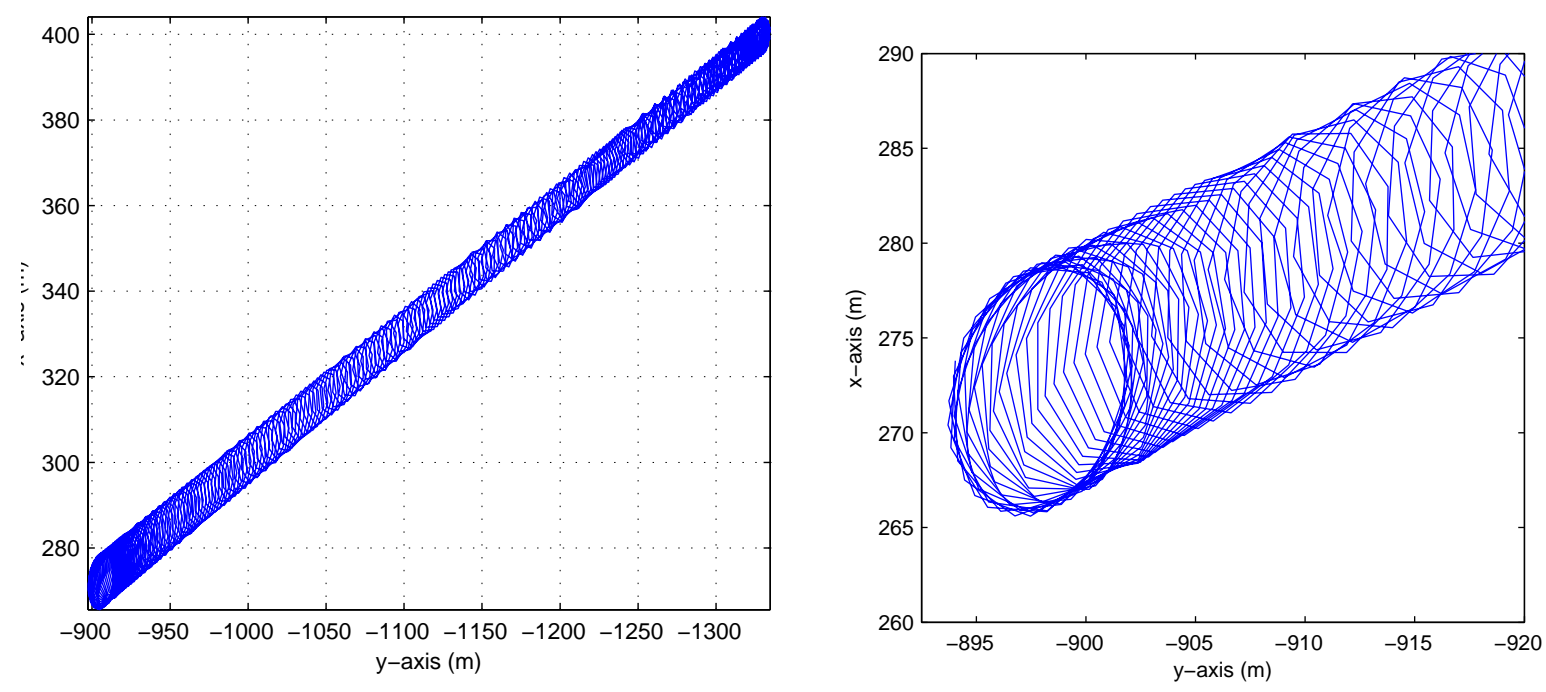

Figure 10: Variation of the equilibrium points with the true anomaly for $A=196 \mathrm{~m}^{2}$ and an ellipsoidal model for the asteroid. Right plot shows a close-up of the left plot.

\section{B. Funnel Reference Orbits}

An alternate approach is to have the mirrors flying in formation with the asteroid, orbiting in tandem around the Sun. The spacecraft have to maintain their relative position with respect to the asteroid in order to keep 
the required power density on the same spot of the surface of the asteroid. Therefore, the formation orbits have to be periodic and in close proximity with low excursion in the relative distance from the asteroid. On the other hand the spacecraft should avoid, as much as possible, to fly in the irregular regions of the gravity field of the asteroid. In addition, should also avoid any impingement with the plume of debris and gas coming from the sublimation of the surface material. In order to design the desired formation orbits, we start by considering the relative equations of motion given in Eq. (10) which use the orbital element differences between a chief orbit (which can be virtual, and is located at the origin of the Hill reference frame) and a spacecraft in the formation. ${ }^{13}$ This is a first approximation of the motion of the spacecraft that does not take into account the gravity field of the asteroid and the solar pressure but it is useful to identify some orbit geometries that answer to our requirements.

The formation orbit can be thought of as an orbit around the Sun with a small offset in the initial position $\delta r_{0}$ and velocity $\delta v_{0}$. This offset can also be expressed as the difference between the orbital parameters of the chief (e.g. Apophis) and the formation. As long as there is no difference in semi-major axes, the two orbits will remain periodic.

$$
\delta \mathbf{k}=\mathbf{k}_{s}-\mathbf{k}_{A}=\left[\begin{array}{llllll}
\delta a & \delta e & \delta i & \delta \Omega & \delta \omega & \delta M
\end{array}\right]
$$

As the mean anomaly is a function of the semi-major axis, the difference in mean anomaly will remain constant through out the orbit so long as $\delta a=0$.

If the optimal thrust direction that maximizes the deviation is along the unperturbed velocity vector of the asteroid, ${ }^{8}$ then the exhaust gases will flow along the $y$-axis of the local Hill reference frame. Therefore, the size of the formation orbits projected in the $x$ - $z$ plane should be maximal. All the requirements on the formation orbits can be formulated in mathematical terms as a multi-objective optimization problem,

$$
\begin{aligned}
& \min _{\delta \mathbf{k} \in D} \min _{f} J_{1}=\delta r \\
& \min _{\delta \mathbf{k} \in D} \min _{f} J_{2}=-\sqrt{x^{2}+z^{2}}
\end{aligned}
$$

subject to the constraint:

$$
C_{\text {ineq }}=\min _{f}\left(\delta r(f)-r_{\mathrm{LIM}}\right)>0
$$

where $r_{\text {LIM }}$ is a minimum-radius sphere imposed to avoid non-linearities in the asteroid gravity field, ${ }^{6}$ and $D$ is the search space for the solution vector $\delta \mathbf{k}$.

The problem in Eqs. (22)-(24) was solved with a hybrid stochastic-deterministic approach based on a multiagent search technique combined with a decomposition of the search space. ${ }^{14,15}$ The result was several groupings, or families, of formation orbits. As can be seen in Fig. 11, the solutions are symmetrically distributed about the 0 -value of the $\delta \mathbf{k}$ parameters. The existence of families can be seen, for example, through $\delta \omega$ and $\delta \Omega$, where for a given input value there are multiple values for the objective functions $J_{1}$ and $J_{2}$. Figure 12 shows the formation orbits in the $\mathcal{A}$ Hill frame. Figure 13 instead shows some particular solutions, close to the limit sphere. These solutions belong to four symmetric families of formation orbits, each one corresponding to a funnel.

\section{Spacecraft Orbital Maintenance}

\section{A. Artificial Equilibrium Points}

Solar pressure depends on the distance from the Sun, therefore, if the size of the mirror is constant, as the asteroid moves around the Sun the force acting to the spacecraft changes with the true anomaly $f$. As a consequence, the position of the equilibrium points changes with time unless the orbit of the asteroid is circular.

Fig. $9 a$ shows, for different attitudes of the mirror, the position of the equilibrium points over a full orbit of the asteroid Apophis. Fig. $9 b$ instead shows the variation of the position of the AEP for a particular attitude of the mirror, over half an orbit. The black dots represent the computed position of the equilibrium 

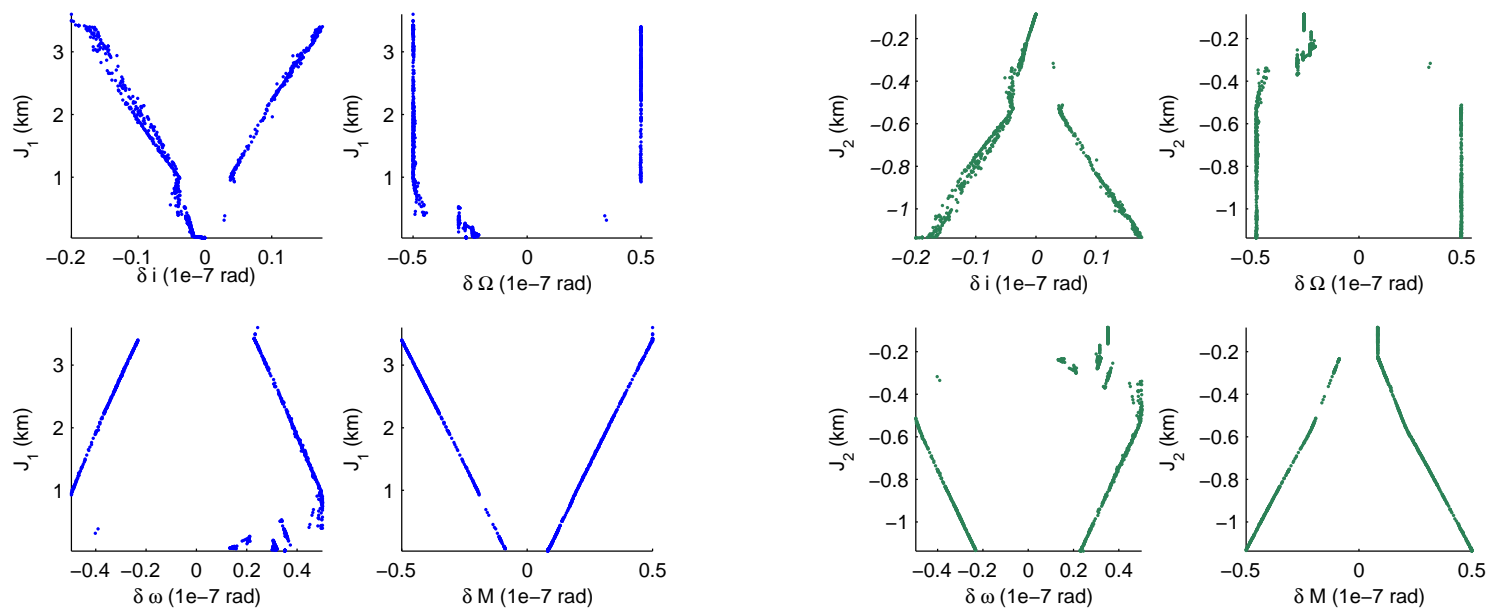

Figure 11: $\delta \mathbf{k}$ parameters of the set of Pareto optimal solutions.

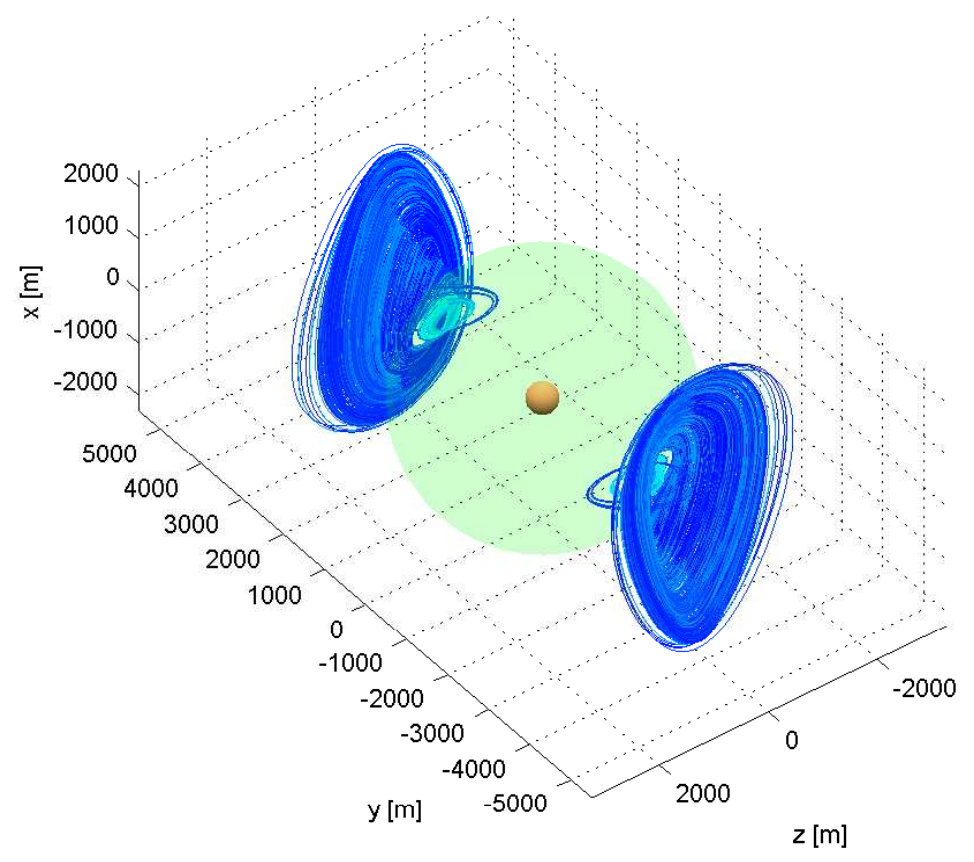

Figure 12: Funnel configuration for the solutions of Eq. 22. 


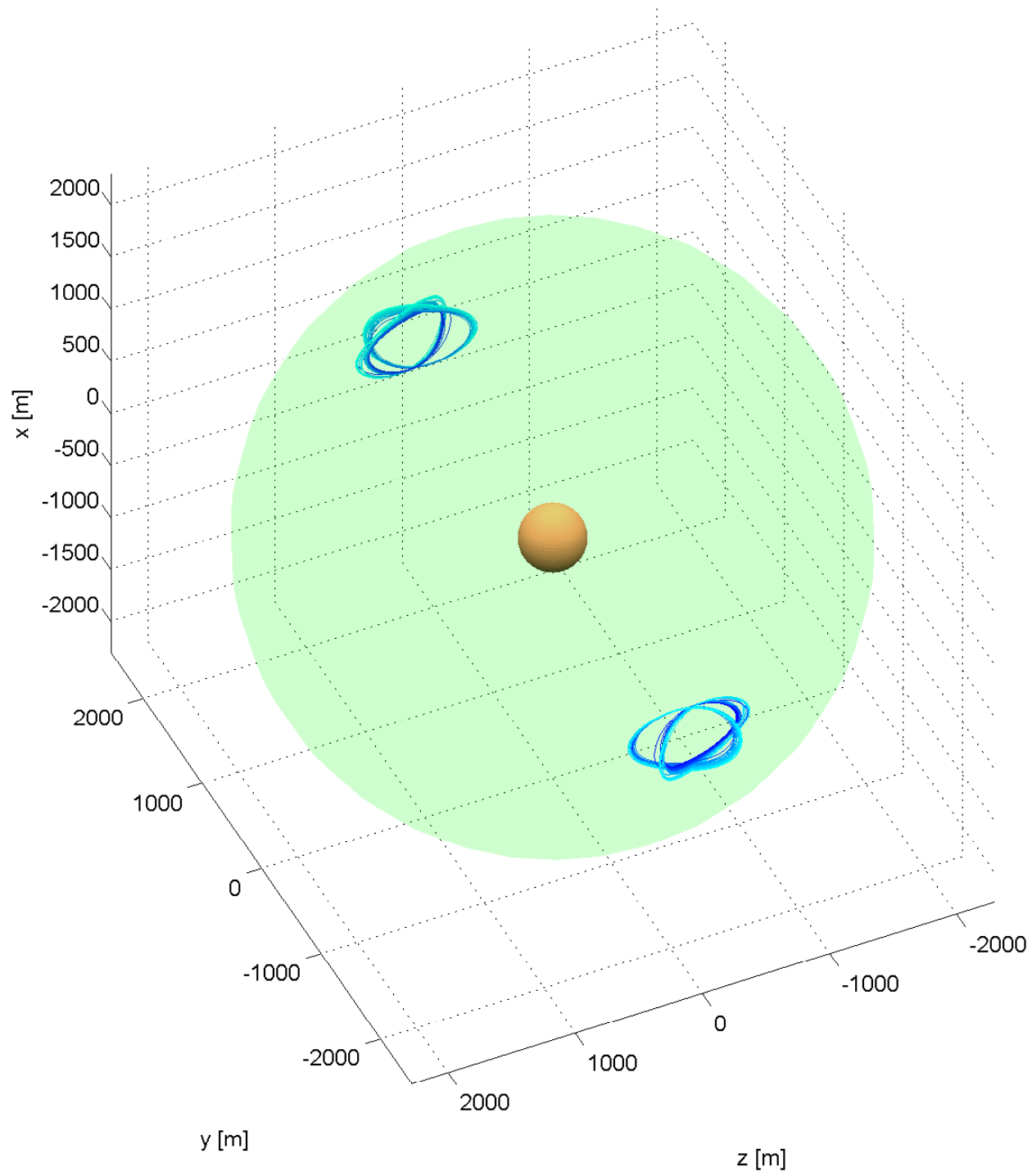

Figure 13: Funnel configuration in close proximity to the NEO, for the solutions of Eq. (22). 
points for an angle $\beta=129^{\circ}$ while the continuous line is given by the following equations,

$$
\begin{aligned}
& x_{\mathrm{AEP}}=\delta r_{\mathrm{AEP}_{0}} \cos \epsilon\left(\frac{1+e \cos f_{0}}{1+e \cos f}\right) \\
& y_{\mathrm{AEP}}=\delta r_{\mathrm{AEP}_{0}} \sin \epsilon\left(\frac{1+e \cos f_{0}}{1+e \cos f}\right)
\end{aligned}
$$

where $\epsilon=\arctan \left(\frac{y_{\mathrm{ARP}}\left(f_{0}\right)}{x_{\mathrm{AEP}}\left(f_{0}\right)}\right)$ is the angular position of the AEP at $f=f_{0}$. Then, the distance of the AEP from the asteroid varies with the following law,

$$
\delta r_{\mathrm{AEP}}=\delta r_{\mathrm{AEP}_{0}} \frac{1+e \cos f_{0}}{1+e \cos f}
$$

Since the AEPs are moving a spacecraft placed at an AEP would depart toward the asteroid or away from the asteroid depending on the initial $f$. In particular, for $f \in[0, \pi]$ the spacecraft would fall toward the asteroid, while for $f \in[\pi, 2 \pi]$ the spacecraft would escape along a radial direction.

We can envisage two strategies to maintain the orbital position of the mirror: we compensate for the between solar pressure and gravity attraction with an active control (low-thrust), or we let the spacecraft drift along the radial direction chasing the position of the equilibrium points.

In order to chase the AEPs the spacecraft has to move with the same kinematics, therefore we can impose the following velocity and acceleration,

$$
\begin{aligned}
\frac{d x}{d t} & =\frac{d\left(\delta r_{\mathrm{AEP}}\right)}{d t} \cos \epsilon & \frac{d y}{d t} & =\frac{d\left(\delta r_{\mathrm{AEP}}\right)}{d t} \sin \epsilon \\
\frac{d^{2} x}{d t^{2}} & =\frac{d^{2}\left(\delta r_{\mathrm{AEP}}\right)}{d t^{2}} \cos \epsilon & \frac{d^{2} y}{d t^{2}} & =\frac{d^{2}\left(\delta r_{\mathrm{AEP}}\right)}{d t^{2}} \sin \epsilon
\end{aligned}
$$

with

$$
\begin{aligned}
\frac{d\left(\delta r_{\mathrm{AEP}}\right)}{d t} & =\frac{\delta r_{\mathrm{AEP}}^{2} e \dot{f} \sin f}{\delta r_{\mathrm{AEP}}} \\
\frac{d^{2}\left(\delta r_{\mathrm{AEP}}\right)}{d t^{2}} & =\frac{e \delta r_{\mathrm{AEP}}}{\delta r_{\mathrm{AEP}}}\left(2 \delta \dot{r}_{\mathrm{AEP}} \dot{f} \sin f+\delta r_{\mathrm{AEP}} \dot{f}^{2} \cos f+\delta r_{\mathrm{AEP}} \ddot{f} \sin f\right)
\end{aligned}
$$

Eqs. (27) and (28) represent an imposed shape to the motion of the spacecraft. If we then substitute Eqs. (25a), (27) and (28) into the dynamic equations in (20) and solve for the controls, we can get the required thrust components to follow the prescribed kinematics. Fig. 14 presents an example of the required thrust profile to maintain a fixed position for an AEP computed at the perihelion. By comparison, Fig. 15 shows the required control profile to make the spacecraft drift following the motion of the AEP for different values of the true anomaly. Both figures assume a spherical model for the asteroid.

As can be seen the control capability required to maintain a fixed position is greater than the one required to chase the AEP. A possible scenario, therefore, is that the swarm can be distributed around the asteroid at different angles $\epsilon$ and the mirrors would move back and forth along the radial directions. As can be seen from the figures, the control authority required to maintain the position of an AEP is several order of magnitude higher, though still very small, than what required to chase the AEP. Figure 16, instead, shows the required control profile to follow the motion of the AEP computed for a spherical asteroid when the gravity field for an elongated body is considered.

\section{B. Funnel Control}

While the design of the artificial equilibrium points accounts for the additional perturbations, the funnel formation design does not. Instead, a control law is required to compensate not only for the solar pressure and third body effects, but also for the constantly changing orbit of the asteroid.

The aim of the control law is to match the relative orbital parameters to those determined by the Funnel orbits, using as feedback the measured position and velocity of the spacecraft relative to the NEO since these values are needed for the tracking and deviation the asteroid. Our initial approach was to use the proximity-quotient, or $Q$-law, originally developed by Petropoulos ${ }^{16}$ for the restricted two-body problem. 


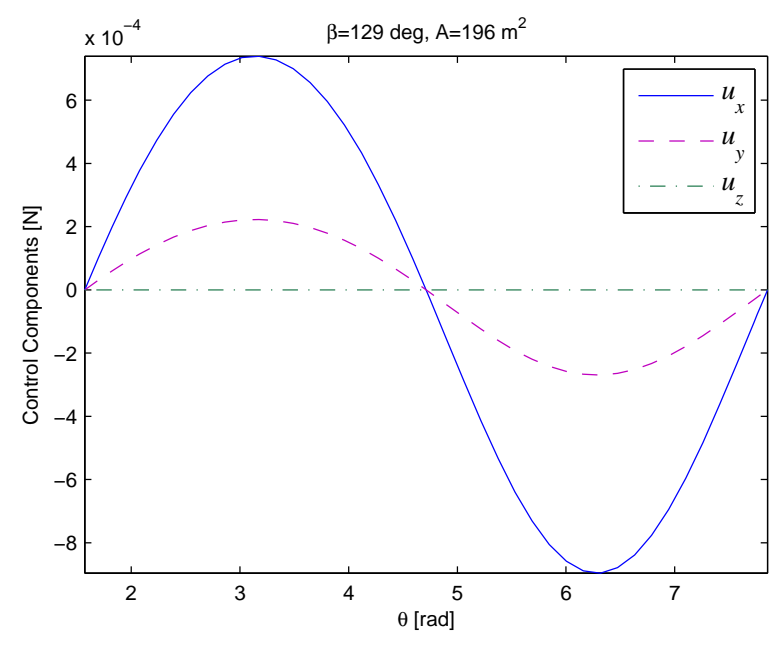

Figure 14: Control profile for orbit maintenance for $\left(A=196 \mathrm{~m}^{2}, \beta=139^{\circ}, f_{0}=\pi\right)$ maintaining a fixed position in the Hill frame $\mathcal{A}$.

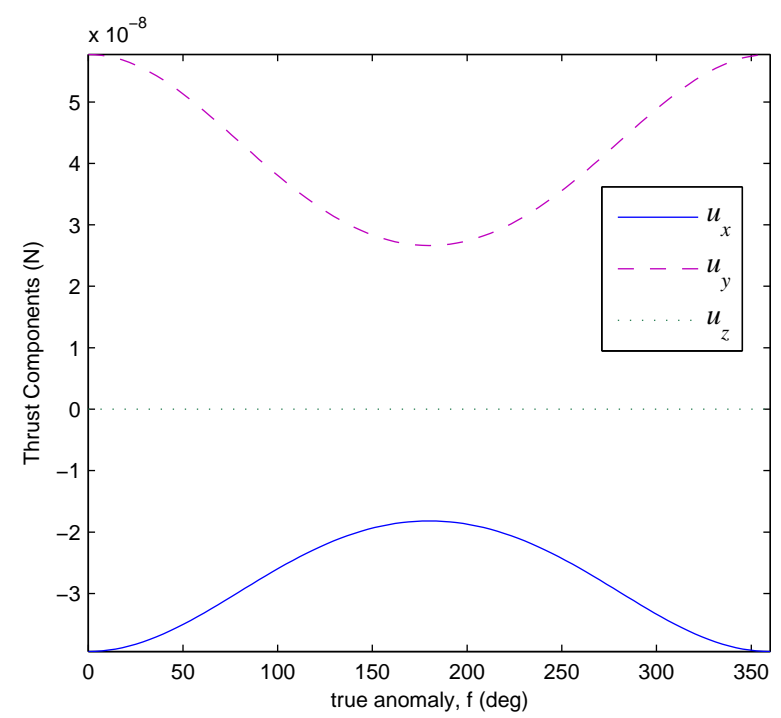

Figure 15: Control profile for orbit maintenance for $\left(A=196 \mathrm{~m}^{2}, \beta=139^{\circ}\right)$ following the motion of the AEP.

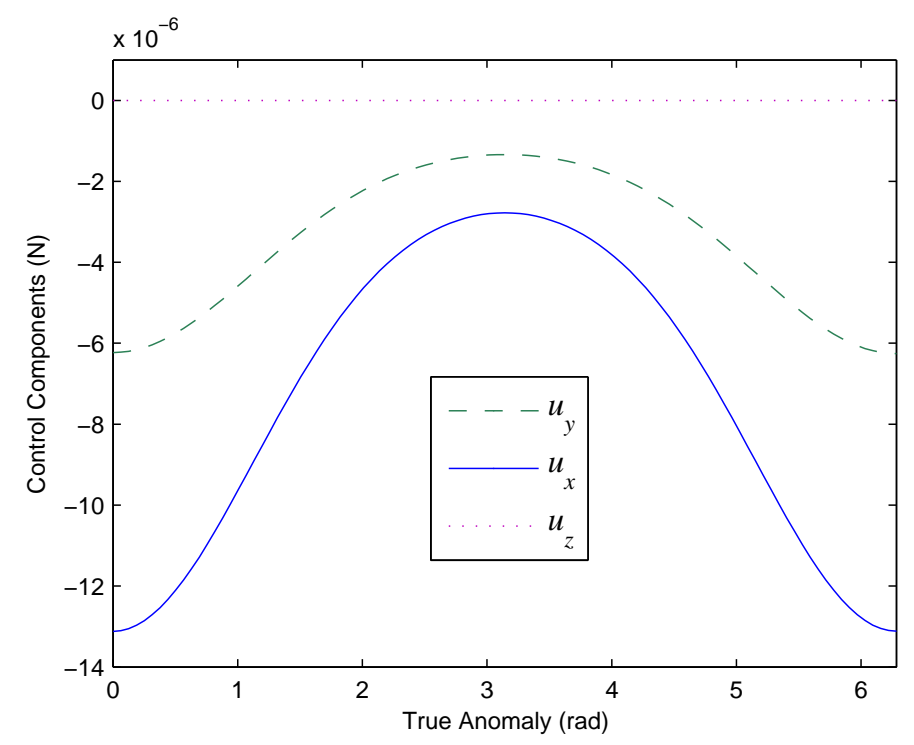

Figure 16: Control required to follow the AEP motion originally computed assuming a spherical asteroid (see Fig. 15) for a non-spherical asteroid. 
The $Q$-law is based on a Lyapunov feedback control law, and calculates the optimal thrust angles based on the proximity to the target orbit (i.e. the difference in the static Keplerian parameters) and the current location of the spacecraft on the orbit (i.e. true anomaly).

$$
Q=\sum_{\delta \mathbf{k}} W_{k}\left(\frac{k_{i}-k_{T}}{k\left(\alpha_{\max }, \beta_{\max }, f_{\max }\right)}\right)^{2} \rightarrow \min (\dot{Q})
$$

where $\dot{k}$ is given by the Gauss equations, setting the thrust angles and orbital location $\alpha=\alpha_{\max }, \beta=$ $\beta_{\max }, f=f_{\max }$ that give the maximum rate of change for each element $a, e, i, \Omega$ and $\omega$. The $Q$-law was developed to provide a first-guess solution for transfers between orbits, not point-to-point, so $M$ (or $f$ ) was left as a free variable. The equations were updated to include the $\dot{M}$ term, and account for SRP and third body effects in the Gauss equations $\dot{\mathbf{k}}$ required for this test case.

However, there were a number of issues that arose: the first was due to the high degree of accuracy need to maintain the funnel orbits. The difference in Keplerian between the NEO and the spacecraft are on the order of $10^{-7}$, and need to remain constant even as the NEO deviates. This resulted in a lot of 'chatter' (over-shooting) around the target orbital elements, due to strong dependance on the time step $\delta t$ and the magnitude of the control (which employed on-off shooting). Even at very small time steps, the magnitude of the over-shotting was too large for the system requirements. The effects of the perturbations are also relatively large (shown in Fig. 17) and need to be compensated for on a continuous basis.
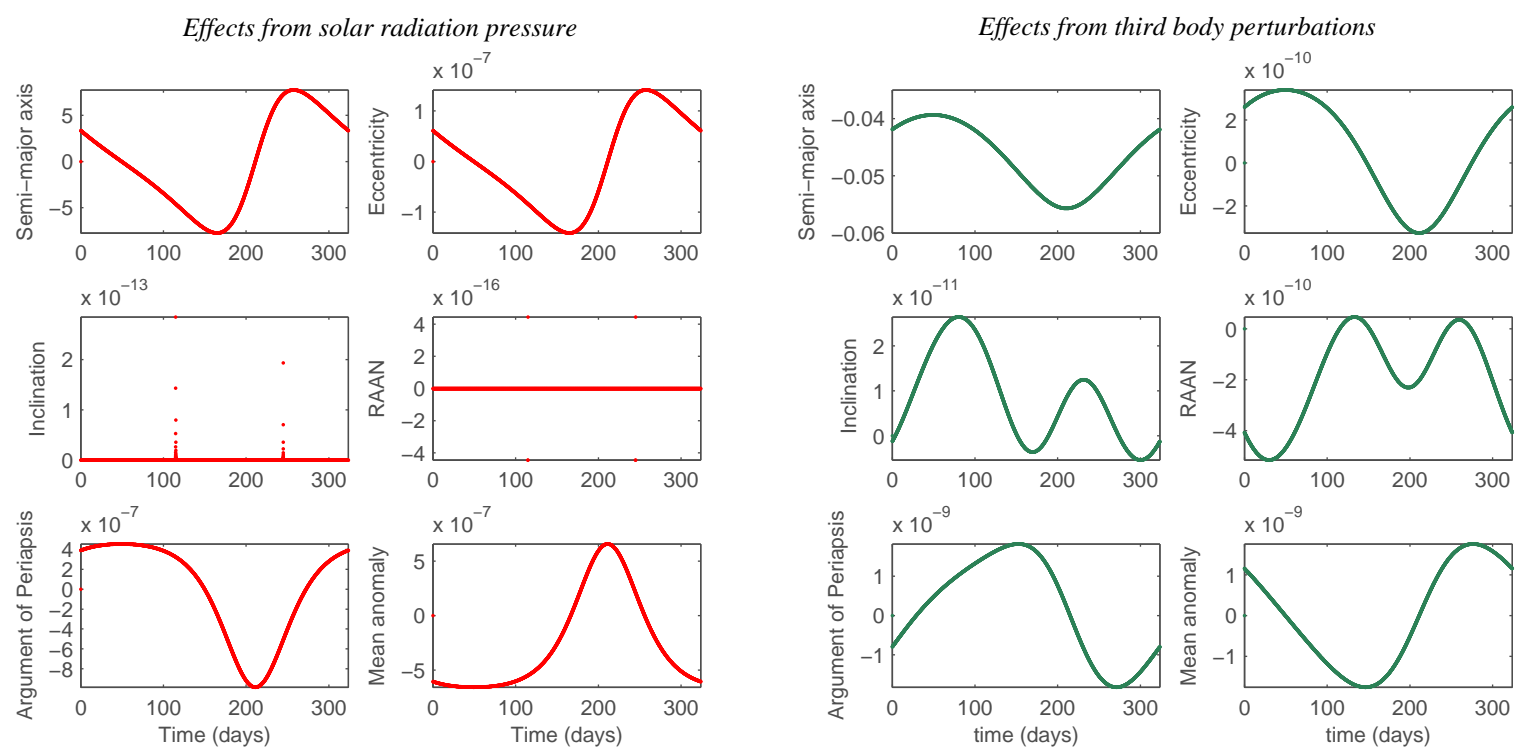

Figure 17: Effects of SRP (left) and third-body (right) on the un-controlled Funnel orbits.

Therefore, an alternative approach was developed to deal with these mission-specific limitations. The first was to switch from minimizing only the thrust angles, to minimizing the components $\left[u_{x}, u_{y}, u_{z}\right]^{T}$ which has the benefit of finding the optimal magnitude for the thrust, as well as the required angles. We considered the new $Q$ function,

$$
Q^{*}=\sum_{j=1}^{6} W_{j}\left(\Delta k_{T, j}-\int_{0}^{\delta t} \frac{d k_{j}}{d t} d \tau\right)^{2}
$$

where $\Delta \mathbf{k}_{T}=\left(\mathbf{k}_{i}-\mathbf{k}_{T}\right)$ is the desired variation of the orbital parameters in the time interval $\delta t$. The function $Q^{*}$ is then minimized with respect to the control components $\left[u_{x}, u_{y}, u_{z}\right]^{T}$ every $\delta t$ units of time.

If we consider that over very small time steps, we can assume as first approximation that the orbital parameters in the Gauss equations are constant, than we can solve directly for control function $\sum W_{j}\left(\Delta k_{T, j}-\frac{d k_{j}}{d t} \delta t\right)^{2}$. Inherently, if the desired change in the $j^{\text {th }}$ element $\left(k_{i, j}-k_{T, j}\right)$ is negative, than the 
rate of change is positive, and vice versa. As such, the control equation will always have a single minimum. Therefore there is no need to minimize the time derivative.

The solution for the control vector $\mathbf{u}_{c}$ is found by using an ordinary least squares fitting ${ }^{17}$ to the linear systems of equations, $\mathbf{A} \mathbf{u}_{c}=\mathbf{b}$. In our case, $\mathbf{A}$ is set equal to the Gauss equations, ${ }^{9}$

$$
\begin{gathered}
\frac{d \mathbf{k}}{d t}=\mathbf{A} \cdot \mathbf{u}_{c} \\
{\left[\begin{array}{c}
\dot{a} \\
\dot{e} \\
\dot{i} \\
\dot{\Omega} \\
\dot{\omega} \\
\dot{M}
\end{array}\right]=\left[\begin{array}{ccc}
\frac{2 a^{2} e \sin f}{h} & \frac{2 a^{2} p}{h r} & 0 \\
\frac{p \sin f}{h} & \frac{(p+r) \cos f+r e}{h} & 0 \\
0 & 0 & \frac{r \cos \theta}{h} \\
0 & 0 & \frac{r \sin \theta}{h \sin i} \\
-\frac{p \cos f}{h e} & \frac{(p+r) \sin f}{h e} & -\frac{r \sin \theta \cos i}{h \sin i} \\
\frac{p \cos f-2 r e}{e \sqrt{a \mu}} & -\frac{(p+r) \sin f}{e \sqrt{a \mu}} & 0
\end{array}\right]\left[\begin{array}{c}
u_{x} \\
u_{y} \\
u_{z}
\end{array}\right]}
\end{gathered}
$$

where $\left[u_{x}, u_{y}, u_{z}\right]^{T}$ are the components of the disturbing acceleration in the radial, transversal and normal directions respectively. The actual Gauss equation for $d M / d t$ also includes a term for the mean motion $n$ to account for the rotation around the Sun. In this case however, we do not want the control to compensate for the nominal motion of the orbit, just those induced by the perturbations and deviation of the asteroid. The mean motion is added to $M^{*}$ after each iteration of the simulation control loop, where $M_{i}=M^{*}+n_{s} \delta t$ (since the nominal rate of the change of the mean anomaly is linear).

The matrix $\mathbf{b}$ is solved by,

$$
\mathbf{b}=\frac{\mathbf{k}_{T}-\mathbf{k}_{i}}{\delta t}-\mathbf{A}\left(\mathbf{u}_{\text {pert }}\right)
$$

Again, this is equivalent to minimizing the quadratic function $\Sigma W_{j}\left(\Delta k_{j}-\Delta k_{T, j}\right)^{2}$ where $\Delta k_{j}$ is the change of the $j^{\text {th }}$ orbital element over time $\delta t$ achieved with Gauss, and $\Delta k_{T, j}$ is the desired change. We use $W_{1}=1 e-3$ to scale down the first row of $\mathbf{A}$ and $\mathbf{b}$ and $W_{2}=1 e 3$ to increase the sensitivity to variations in eccentricity. This was just a preliminary attempt to find a good set of weights, a more accurate tuning of the $W_{j}$ could lead to better results.

The force equations for the solar pressure given in Eq. (9) can be rewritten in terms of the orbital elements of the formation, and the orbital element differences compared with the asteroid. All the equations are relative to the $\mathcal{S}$ Hill frame. The magnitude of the disturbing acceleration due to the solar pressure on the parabolic and directional mirrors respectively are given as,

$$
\begin{aligned}
S_{p} & =\frac{2 P_{r} \eta_{e f f} A_{p}}{m_{s}} \\
S_{d} & =\frac{2 P_{r} \eta_{e f f}}{m_{s}} \cos ^{2} \alpha\left(A_{p}-\eta_{\text {eff }} A_{d}\right)
\end{aligned}
$$

with

$$
\cos ^{2} \alpha=\frac{r_{\mathrm{A}}^{2}}{|2 \Gamma \delta r|}(\cos (\delta \theta-\theta) \sin \delta \Omega \sin i+\varpi \sin (\delta \theta-\theta))^{2}
$$

where $\alpha$ is the angle of reflection, $\eta_{\text {eff }}=\eta_{\text {eff }}=\eta_{\text {eff }}=90 \%, \varpi, \xi, \varrho, \zeta$ are defined in Eq. (11), and lastly

$$
\Gamma=\delta r+r_{s}-r_{\mathrm{A}}(\cos \delta \Omega \cos (\delta \theta-\theta) \cos \theta+\cos (\delta i-i) \cos \theta \sin \delta \Omega \sin (\delta \theta-\theta)+\zeta \sin \theta)
$$

The unit vector $\hat{\mathbf{s}}_{d}$ gives the direction of the net force due to the SRP on the secondary directional mirror (i.e. $\left.\mathbf{F}_{2}+\mathbf{F}_{3}\right)$.

$$
\hat{\mathbf{s}}_{d}=\frac{\mathbf{n}_{d}}{\left\|\mathbf{n}_{d}\right\|}
$$

$$
\left.\mathbf{n}_{d}=\left[\begin{array}{l}
-\sqrt{\frac{\Gamma}{2 \delta r}}\left(r_{s}+r_{\mathrm{A}} \varrho \cos \theta+r_{\mathrm{A}} \zeta \sin \theta\right) \\
r_{\mathrm{A}}(\cos i \cos (\delta \theta-\theta) \cos \theta \sin \delta \Omega+\xi \cos \theta \sin (\delta \theta-\theta)-\varrho \sin \theta) \\
r_{\mathrm{A}}(\cos (\delta \theta-\theta) \sin \delta \Omega \sin i+\varpi \sin (\delta \theta-\theta))\left(\sqrt{\frac{2 \Gamma}{\delta r}}+r\left(r_{s}+r_{\mathrm{A}} \varrho \cos \theta+r_{\mathrm{A}} \zeta \sin \theta\right)\right)
\end{array}\right]\right]^{\mathcal{S}}
$$


Since the primary mirror is always aligned with the Sun, the direction of net acceleration is simply $\hat{\mathbf{s}}_{p}=$ $[1,0,0]^{\mathcal{S}}$.

Since the effects of the asteroid's gravity field outside the imposed limiting sphere are relatively linear, ${ }^{6}$ and much less compared to those due the solar radiation pressure, the asteroid is treated, as a first approximation, as a point mass with $\mu_{\mathrm{A}}=1.8016 \mathrm{e}-9 \mathrm{~km}^{3} / \mathrm{s}^{2}$. Adding the perturbing acceleration due to a third body point mass, the perturbing control vector becomes,

$$
\mathbf{u}_{\text {pert }}=\left(S_{p} \hat{\mathbf{s}}_{p}+S_{d} \hat{\mathbf{s}}_{d}\right)-\frac{\mu_{\mathrm{A}}}{\delta r^{3}} \delta \mathbf{r}
$$

The individual steps of the simulation routine are described in Algorithm 1.

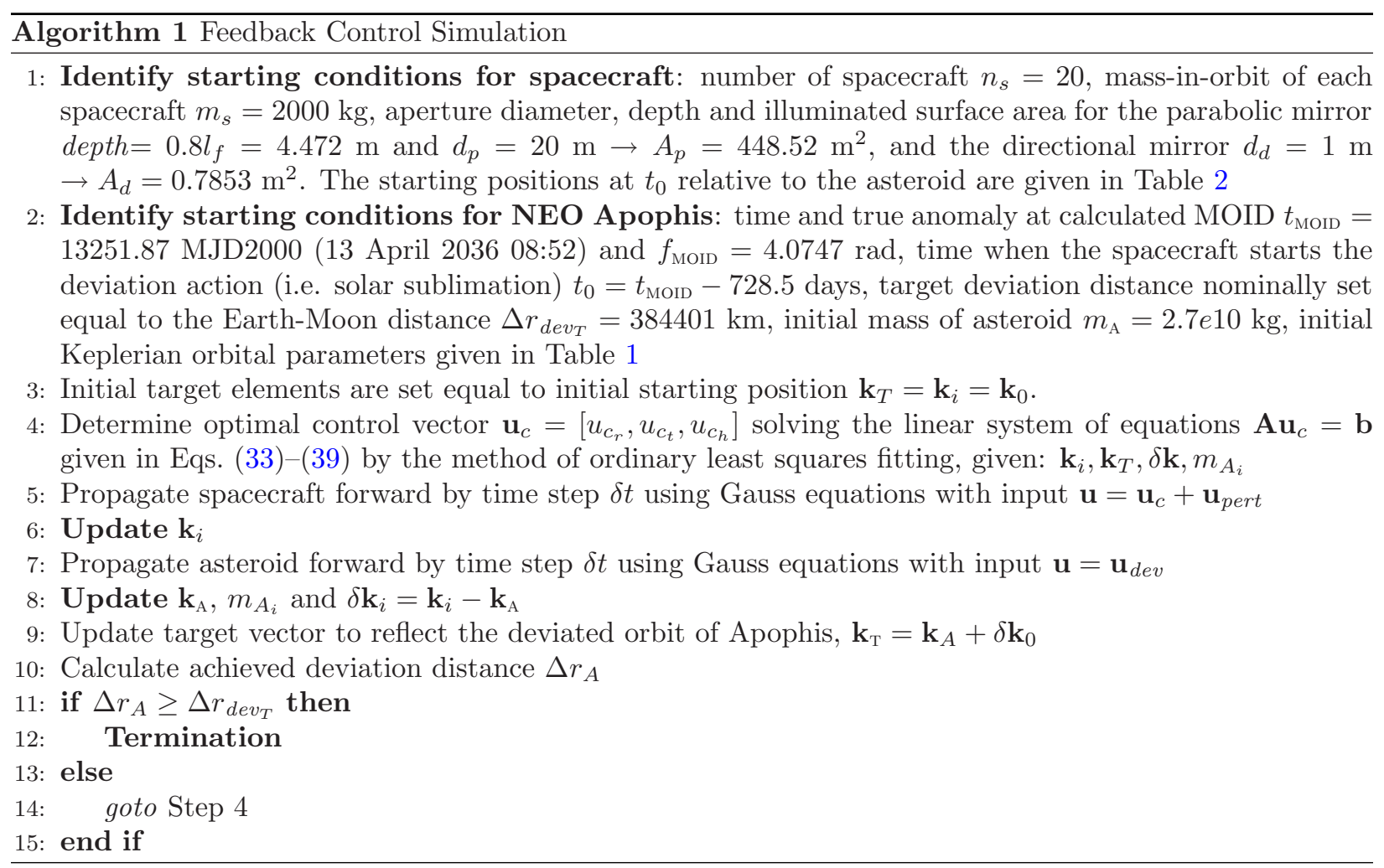

Simulations were run at two different time steps, $\delta t=[1,10] \mathrm{s}$ using a test orbit chosen out of the set of Pareto optimal Funnel solutions. Table 2 gives the initial state vector for the test orbit. The thrust leg of the mission was started 13 April 2031 - 5 years before the first potential impact. The required thrust duration is 552.30 days to reach a deviation distance of $384400 \mathrm{~km}$ (equal to the Earth-Moon distance) in 2036. Figure 18 shows the deviation of the orbital elements of the NEO during the thrust duration. For the control of the orbit, Figs. 19, 21 show the difference in Keplerian parameters between the actual and target states during the part of the thrust leg, and Figs. 20, 22 the corresponding components of the low-thrust control acceleration.

\section{Conclusion}

In this paper we presented an analysis of the proximal motion and station-keeping for a set of mirrors operating in the vicinity of an asteroid. Two configurations for the mirrors were analyzed and for each one a different strategy for orbit maintenance was considered. In particular, the dual-mirror configuration led to the definition of a particular set of formation orbits composing two symmetric funnels with the principal axis aligned with the $y$-axis of the Hill reference frame. These funnel orbits allow the spacecraft to have a very good visibility of the target spot on the surface of the asteroid and at the same time allow room for the plume of gas to flow with minimal impingement. The funnel orbits are located outside a limiting sphere where the gravity field of the asteroid can be considered homogenous. This limit sphere imposes requirements on the pointing accuracy and focusing capabilities of the mirror assembly. The orbital maintenance strategy 
Table 2: Optimized initial conditions for spacecraft at $t_{0}$.

\begin{tabular}{cccc}
$J_{1}(\mathrm{~m})$ & $J_{2}(\mathrm{~m})$ & Element in $\delta \mathbf{k}_{0}$ & Value $(\mathrm{rad})$ \\
\hline 88.8845 & -241.1802 & $\delta a$ & 0 \\
& & $\delta e$ & $6.9071 \mathrm{e}-012$ \\
& & $\delta i$ & $-1.7903 \mathrm{e}-009$ \\
& & & \\
& & $\delta \omega$ & $-2.3827 \mathrm{e}-008$ \\
& & & $3.1574 \mathrm{e}-008$ \\
& & $8.9855 \mathrm{e}-009$ \\
\hline
\end{tabular}
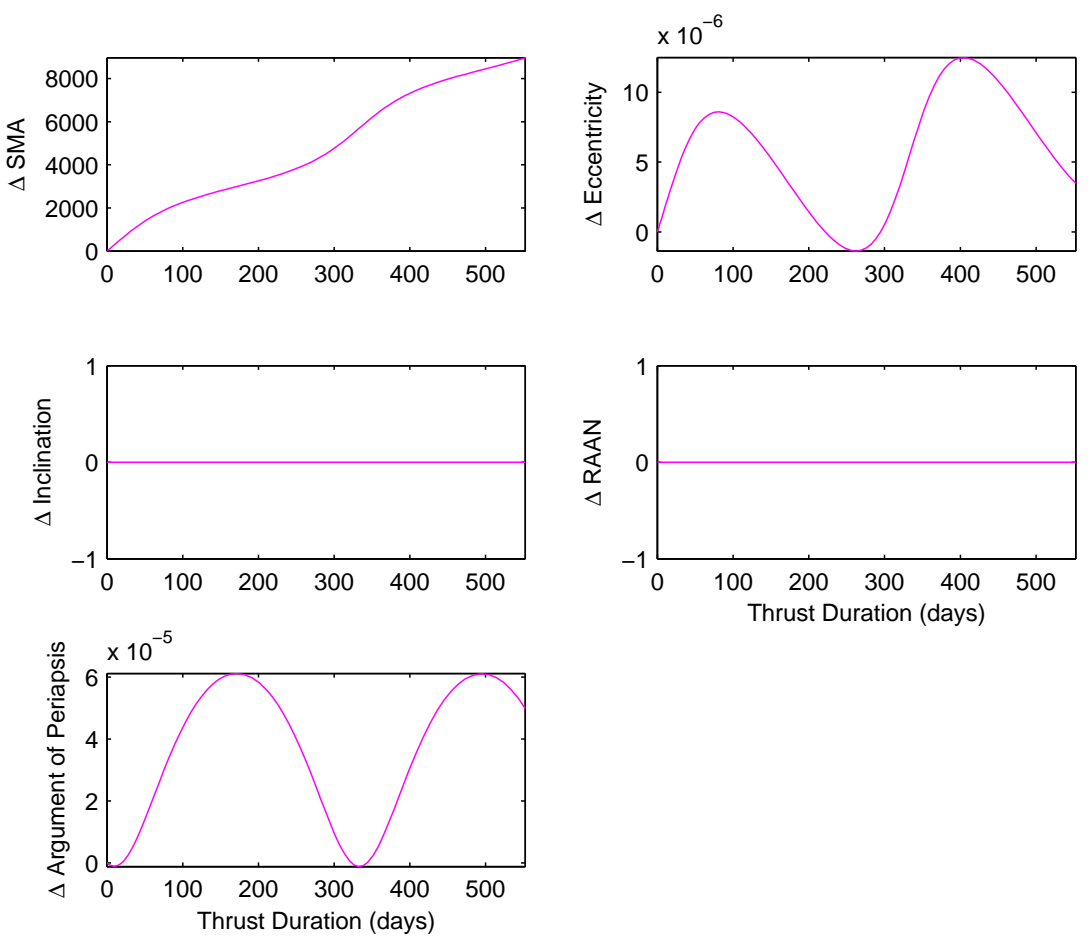

Figure 18: Deviation of Keplerian elements for Apophis, with a warning time of 5 years before the 2036 potential impact for a deviation distance of $384400 \mathrm{~km}$ in 2036 . 

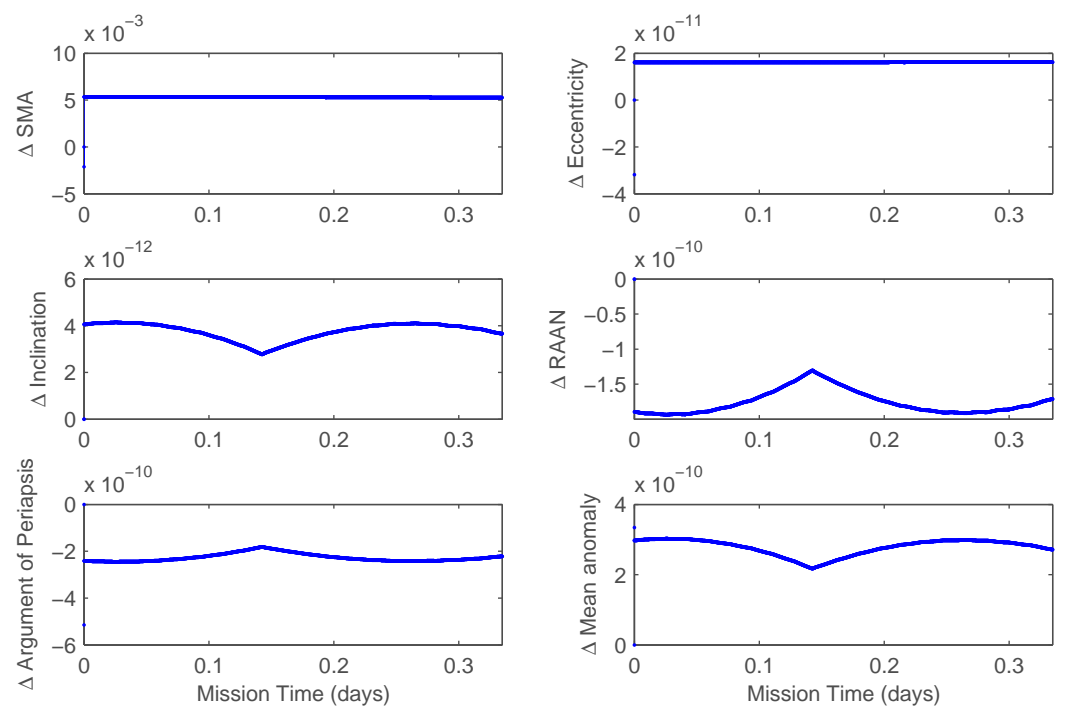

Figure 19: Difference between current state $\mathbf{k}_{i}$ and target state $\mathbf{k}_{T}$ with a time step $\delta t=1 \mathrm{~s}$ (plot units are in $\mathrm{km}, \mathrm{rad})$.
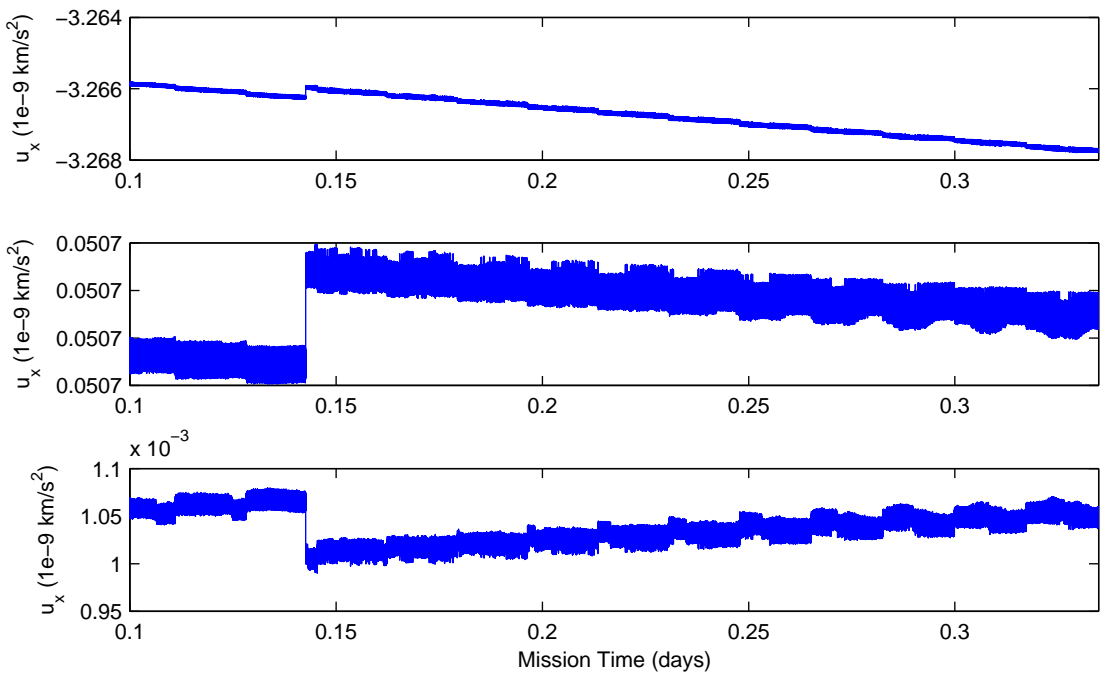

Figure 20: Control components for $\delta t=1 \mathrm{~s}$ 

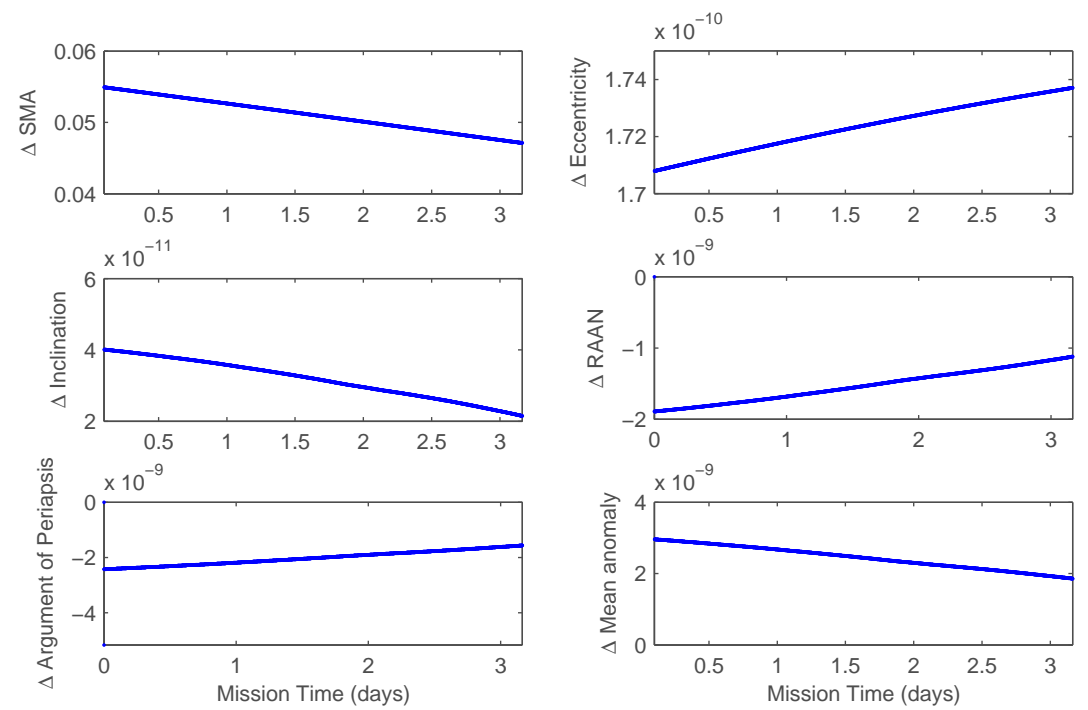

Figure 21: Difference between current state $\mathbf{k}_{i}$ and target state $\mathbf{k}_{T}$ with a time step $\delta t=10 \mathrm{~s}$ (plot units are in $\mathrm{km}, \mathrm{rad})$.
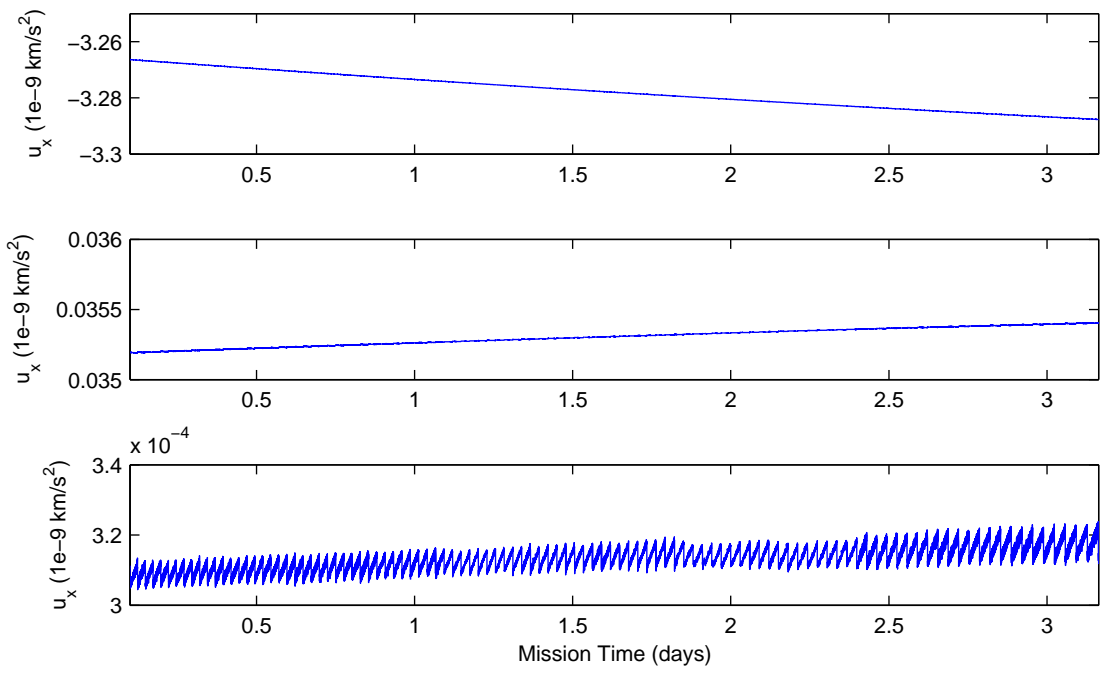

Figure 22: Control components for $\delta t=10 \mathrm{~s}$ 
is based on the computation of the control components that minimize the difference between the current and target value of the relative Keplerian elements. The control compensated for two sources of perturbations: solar radiation pressure due to the large surface area of the mirror assembly and third body effects due to proximity of Apophis, and for the constantly deviating orbit of the NEO. The decrease in mass of Apophis due to the solar sublimation was also accounted for. The control is only required for the duration of the mission, in this case to amount of time necessary to achieve a deviation distance equal to the Earth-Moon separation. However the short simulation duration in the figures are due to limited computer resources. From these preliminary results we can see that the control applied with a time step of $1 \mathrm{~s}$ can maintain the orbit relatively stable and close to the nominal one. With a time step of $10 \mathrm{~s}$ the control overshoots and then slowly tries to return to the nominal orbit. A full integration of Gauss' equations in the interval of time in which the control is applied, is expected to give a better prediction of the control components leading to a more accurate control of the orbital elements.

A second option considered a single-mirror configuration. For this option, the mirror can be placed at artificial equilibrium points highly inclined over the $y$-axis of the Hill frame. From this position the spacecraft sees the target spot from a high angle, however AEPs can be found that allow the spacecraft to maintain a reasonable size of the spot area. A control strategy was proposed that allows the spacecraft to oscillate in a confined region in the proximity of the asteroid with a very low control thrust. Even adding the effect of the gravity field of an elongated body, the magnitude of the required control thrust remains limited. The low level of thrust would suggest the use of FEEP engines, which would lead to a minimal propellant consumption even over long operation times.

\section{Acknowledgments}

This research is partially supported by the ESA/Ariadna Study Grant AO/1-5387/07/NL/CB. The authors would like to thank Dr. Leopold Summerer of the ESA Advanced Concepts Team for his support.

\section{References}

\footnotetext{
${ }^{1}$ Colombo, C., Sanchez Cuartielles, J. P., Vasile, M., and Radice, G., "A comparative assessment of different deviation strategies for dangerous NEO," 56th International Astronautical Conference Proceedings, Valencia, Spain, October 2006.

${ }^{2}$ Lunan, D., "Need we protect Earth from space objects and if so, how?" Space Policy, Vol. 8, No. 1, 1992, pp. 90-91.

${ }^{3}$ Melosh, H. J. and Nemchinov, I. V., "Solar asteroid diversion," Nature, Vol. 366, No. 366, 1993, pp. 21-22.

${ }^{4}$ Kahle, R., Kührt, E., Hahn, G., and Knollenberg, J., "Physical limits of solar collectors in deflecting Earth-threatening asteroids," Aerospace Science and Technology, Vol. 10, 2006, pp. 253-263.

${ }^{5}$ Maddock, C., Sanchez Cuartielles, J. P., Vasile, M., and Radice, G., "Comparison of single and multi-spacecraft configurations for NEA deflection by solar sublimation," American Institute of Physics - New Trends in Astrodynamics and Applications III, edited by E. Belbruno, Vol. 886, 2007, pp. 303-316.

${ }^{6}$ Sanchez Cuartielles, J. P., Colombo, C., Vasile, M., and Radice, G., "A Multi-criteria Assessment of Deflection Methods for Dangerous NEOs," American Institute of Physics - New Trends in Astrodynamics and Applications III, edited by E. Belbruno, Vol. 886, 2007, pp. 317-333.

${ }^{7}$ NASA Near Earth Object Program, "99942 Apophis (2004 MN4) Impact Risk," Online Database, http://neo.jpl.nasa.gov/risk/a99942.html, August 2008.

${ }^{8}$ Vasile, M. and Colombo, C., "Optimal Impact Strategies for Asteroid Deflection," Journal of Guidance, Control and Dynamics, Vol. 31, No. 4, July-August 2008, pp. 858-872. 1999.

${ }^{9}$ Battin, R. H., An Introduction to the Mathematics and Methods of Astrodynamics, AIAA Education Series, revised ed.,

${ }^{10}$ Sanchez Cuartielles, J. P., Colombo, C., Vasile, M., and Radice, G., "Multi-criteria Comparison among Several Mitigation Strategies for Dangerous Near Earth Objects," Journal of Guidance, Control and Dynamics, To appear, Accepted 2008.

${ }^{11} \mathrm{Hu}$, W. and Scheeres, D. J., "Spacecraft Motion About Slowly Rotating Asteroids," Journal of Guidance, Control and Dynamics, Vol. 25, No. 7, July 2002, pp. 765-775.

${ }^{12}$ Rossi, A., Marzari, F., and Farinella, P., "Orbital Evolution Around Irregular Bodies," Earth, Planets, Space, Vol. 51, 1999, pp. 1173-1180. 2003.

${ }^{13}$ Schaub, H. and Junkins, J. L., Analytical mechanics of space systems, AIAA Education Series, Virginia, U.S.A., 1st ed.,

${ }^{14}$ Maddock, C. and Vasile, M., "Design of optimal spacecraft-asteorid formations through a hybrid global optimization approach," Journal of Intelligent Computing and Cybernetics, Vol. 1, No. 2, 2008, pp. 239-268.

${ }^{15}$ Vasile, M., Multi-Objective Memetic Algorithms, chap. Hybrid Behavioral-Based Multiobjective Space Trajectory Optimization, Studies in Computational Intelligence, Springer, 2008.

${ }^{16}$ Petropoulos, A., "Simple control laws for low-thrust orbit transfers," AIAA/AAS Astrodynamics Specialists' Conference, AIAA, Montana, U.S.A., August 2003.
} 
${ }^{17}$ Strang, G., Introduction to Applied Mathematics, Wellesley-Cambridge, 1986. 